\title{
Pd(0)-Catalyzed Amination in the Synthesis of Bicyclic Compounds Comprising Triazacycloalkane and Fluorophore Moieties
}

\author{
Alexei D. Averin, ${ }^{\mathrm{a}, \mathrm{b} @ ~ N a t a l i y a ~ M . ~ C h e r n i c h e n k o, ~}{ }^{a}$ Vadim N. Shevchuk, ${ }^{\mathrm{a}}$ \\ Olga A. Maloshitskaya, ${ }_{1}{ }^{\text {Franck Denat }}{ }^{\mathrm{c}}$ and Irina P. Beletskaya ${ }^{\mathrm{a}, \mathrm{b}}$ \\ ${ }^{a}$ Lomonosov Moscow State University, Department of Chemistry, 119991 Moscow, Russia \\ ${ }^{\mathrm{b}}$ A.N. Frumkin Institute of Physical Chemistry and Electrochemistry RAS, 119991 Moscow, Russia \\ 'ICMUB UMR6302, CNRS, Université Bourgogne Franche-Comté, 21000 Dijon, France \\ ${ }^{\circledR}$ Corresponding author E-mail: alexaveron@yandex.ru
}

\begin{abstract}
Direct introduction of one dansyl fluorophore group into the molecule of 1,4,7-triazacyclononane (TACN) and 1,5,9-triazacyclododecane (TACD) was shown to be possible followed by the modification of triazacycles with two bromobenzyl substituents. The resulting compounds were used in the Pd(0)-catalyzed macrocyclization reaction with 1,13-diaza-4,7,10-trioxatridecane to provide corresponding cryptands. The synthesis of bicyclic derivatives of TACN bearing naphthyl and acridinyl fluorophore groups was accomplished via an alternative procedure using aminal-protected TACN. The possibility to form tetracyclic compounds starting from N,N',N"'-tris(3-bromobenzyl) substituted TACN was demonstrated. Three cryptands bearing dansyl fluorophore were tested as potential fluorescent chemosensors for metal cations and were found to act as molecular fluorescent probes for Cu(II) and Al(III) by total emission quenching in the presence of these cations.
\end{abstract}

Keywords: Pd Catalysis, triazacycles, amination, cryptands, fluorescence.

\section{Pd(0)-Катализируемое аминирование в синтезе бициклических соединений, содержащих триазациклоалкановые и флуорофорные фрагменты}

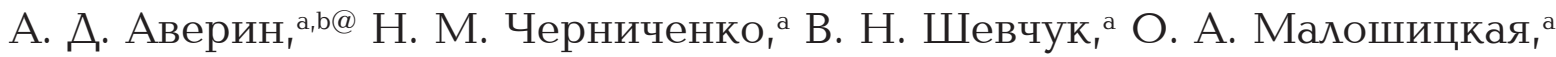 \\ Ф. Аена, ${ }^{c}$ И. П. Белецкая ${ }^{\mathrm{a}, \mathrm{b}}$
}

${ }^{\mathrm{a}}$ Московский государственньй университет им. М.В. Ломоносова, Химический факультет, 119991 Москва, Россия

' Институт физической химии и электрохимии им. А.Н. Фрумкина РАН, 119071 Москва, Россия

'Институт молекулярной химии Университета Бургундии и Франш-Конте, 21000 Дижон, Франция

@E-mail: alexaveron@yandex.ru

\begin{abstract}
Показана возможность прямого введения одной дансильной флуорофорной группьл в молекулу 1,4,7-триазаииклононана (ТАЦН) и 1,5,9-триазациклододекана (ТАЦД) с последующей модификаичей триазациклов двумя бромбензильными заместителями. Полученные соединения использованы в Pd(0)-катализируемой макроциклизации с 1,13-диаза-4,7,10-триоксатридеканом с образованием соответствующих криптандов. С использованием альтернативного подхода через ТАЦН с аминальной защчитой были синтезированы бицикль, содержащче нафтильный и акридинильный флуорофоры. Продемонстрирована возможность получения тетрациклических соединений различного строения исходя из N,N',N”-трис(3-бромбензил) замещенного ТАЦН. Три криптанда исследованы в качестве флуоресиентных детекторов катионов металлов и показана их возможность выступать в качестве молекулярных проб на катионы Си(II) и Al(III) за счет полного тушения флуоресценции в присутствии данных металлов.
\end{abstract}

Ключевые слова: Рd Катализ, триазациклы, аминирование, криптанды, флуоресценция. 


\section{Introduction}

One of the important tasks of the modern organic chemistry is the search for new chemosensors able of selective detection of metal cations. Chemosensors exploiting fluorescence for producing analytical response possess many favorable features like higher sensitivity and selectivity compared to colorimetric detectors, possibility to create ratiometric sensors. At present time many examples of the derivatives of crown and azacrown ethers, ${ }^{[1]}$ calix[4] arene, ${ }^{[2]}$ and thiacalix [4] arene, ${ }^{[3]}$ tetraazamacrocycles ${ }^{[4]}$ bearing fluorescent groups have been described in literature. A special interest paid to 1,4,7-triazacyclononane (TACN) and compounds produced on its basis arises from the application of their metal complexes primarily in the synthesis of bifunctional radiopharmaceuticals capable of selective coordination of ${ }^{111} \mathrm{In},{ }^{67 / 68} \mathrm{Ga},{ }^{64 / 67} \mathrm{Cu},{ }^{90} \mathrm{Y}$, and ${ }^{99 \mathrm{~m}} \mathrm{Tc}$ cations ${ }^{[5]}$ which often possess additional chelating carboxylate and phosphonate arms. ${ }^{[6]}$ Till now the data on the fluorescent derivatives of TACN used as chemosensors are quite scarce. One can cite the reports on the tridansyl (dansyl=5-dimethylamino-1-sulphonylnaphthalene) substituted triazacyclononane, ${ }^{[7]}$ benzothiazole derivative bearing two additional pyridylmethyl arms, ${ }^{[8]}$ TACN modified with pyrene fluorophore. ${ }^{[9]}$ The tridansyl derivative was used as a fluorescent chemosensor for $\mathrm{Ag}(\mathrm{I})$ and $\mathrm{Cu}(\mathrm{II})$ cations, and the compounds bearing this fluorophore group attached to various ionophores were described for the detection of $\mathrm{Hg}(\mathrm{I}),{ }^{[10]}$ $\mathrm{Cu}(\mathrm{II}),{ }^{[11]} \mathrm{Pb}(\mathrm{II}),{ }^{[12]} \mathrm{Tl}(\mathrm{I}), \mathrm{Cs}(\mathrm{I}),{ }^{[13]}$ and $\mathrm{Hg}(\mathrm{I}){ }^{[14]}$ cations. No information can be found about the synthesis of bicyclic compounds comprising TACN unit and their application for detecting metal ions though cryptand-like structures are of special interest due to their special character of binding cations. In our previous research we successfully applied $\operatorname{Pd}(0)$-catalyzed amination reaction to the synthesis of macrocyclic ${ }^{[15]}$ and polymacrocyclic ${ }^{[16]}$ compounds and began the studies of their coordination abilities towards metal cations. The first examples of the porphyrin-derived conjugates were shown to be promising fluorescent chemosensors for $\mathrm{Cu}(\mathrm{II})$ cations. ${ }^{[17]}$ In this work we develop this approach in the synthesis of bicyclic compounds comprising triazacycloalkane units and fluorophore groups.

\section{Experimental}

NMR spectra were registered using Bruker Avance 400 spectrometer, MALDI-TOF spectra were obtained with Bruker Autoflex II spectrometer using 1,8,9-trihydroxyanthracene as matrix and PEGs as internal standards. UV-Vis spectra were recorded with Agilent Cary 60 spectrophotometer in $\mathrm{MeCN}$, spectra of fluorescence were obtained with Horiba Jobin Yvon Fluoromax 2 spectrofluorometer in acetonitrile (UHPLC grade). 1,4,7-Triazacyclononane (TACN) and 1,5,9-triazacyclododecane (TACD) were provided by CheMatech Co (Dijon, France), 3 - and 4-bromobenzyl bromides, trioxadiamine, 1-aza-15-crown-5 ether, tris(2-aminoethyl)amine, benzaldehyde, dansyl chloride (dansyl=5-dimethylamino-1-sulphonylnaphthalene), 2-bromoand 2-(chloromethyl)naphthalene, 9-(bromomethyl)acridine, rac-BINAP and DavePhos ligands, sodium tert-butoxide, were purchased from Sigma-Aldrich Co and used without further purification, $\mathrm{Pd}(\mathrm{dba})_{2}$ was synthesized according to the method described. ${ }^{[18]}$ Acetonitrile of UHPLC grade was used without additional purification, dioxane was successively distilled over $\mathrm{NaOH}$ and sodium. Chloroform was distilled over $\mathrm{P}_{2} \mathrm{O}_{5}$, dichloromethane was distilled over $\mathrm{CaH}_{2}$, methanol was used freshly distilled.

Method for the synthesis of dansyl derivatives of triazacycloalkanes 3, 4. A one-neck flask equipped with a magnetic stirrer was charged with TACN (1) or TACD (2) (2-3 mmol) in acetonitrile, potassium carbonate and the diluted solution of dansyl chloride in acetonitrile was added slowly dropwise during $c a 4 \mathrm{~h}$ The residue was filtered off, washed with chloroform (in the case of TACN derivatives) or dichloromethane (in the case of TACD derivatives) $(5 \mathrm{ml})$, the combined organic fractions were evaporated in vacuo and chromatographed on silica gel using a sequence of eluents: $\mathrm{CHCl}_{3}, \mathrm{CHCl}_{3}-\mathrm{MeOH}$ 100:1-2:1, $\mathrm{CHCl}_{3}-\mathrm{MeOH}-\mathrm{NH}_{3 \text { aq }}$ 100:20:1-100:20:2 (for TACN derivative) or $\mathrm{CH}_{2} \mathrm{Cl}_{2}, \mathrm{CH}_{2} \mathrm{Cl}_{2}-$ $\mathrm{MeOH}$ 100:1-2:1 (for TACD derivative).

5-(1,4,7-Triazacyclononan-1-ylsulfonyl)- $N, N$-dimethylnaphthalene-1-amine (3). Obtained from TACN (1) $(387 \mathrm{mg}, 3 \mathrm{mmol})$, dansyl chloride $(540 \mathrm{mg}, 2 \mathrm{mmol})$ in the presence of potassium carbonate $(2070 \mathrm{mg}, 15 \mathrm{mmol})$ in $105 \mathrm{ml}$ acetonitrile. Eluent: $\mathrm{CHCl}_{3}$ $\mathrm{MeOH}-\mathrm{NH}_{3 \text { aq }}$ 100:20:1-100:20:2, yellow viscous oil. Yield $493 \mathrm{mg}$ (68\%). m/z (MALDI-TOF) found: $363.1815 . \mathrm{C}_{18} \mathrm{H}_{27} \mathrm{~N}_{4} \mathrm{O}_{2} \mathrm{~S}$ requires $363.1849[\mathrm{M}+\mathrm{H}]^{+}$. ${ }^{1} \mathrm{H} \mathrm{NMR}\left(\mathrm{CDCl}_{3}, 298 \mathrm{~K}\right) \delta_{\mathrm{H}}$ ppm: $1.94(2 \mathrm{H}$, br. s, NH), $2.82\left(6 \mathrm{H}, \mathrm{s}, \mathrm{CH}_{3}\right), 2.84\left(4 \mathrm{H}, \mathrm{s}, \mathrm{CH}_{2} \mathrm{NH}\right), 3.02-3.06(4 \mathrm{H}$, $\left.\mathrm{m}, \mathrm{CH}_{2} \mathrm{NH}\right), 3.33-3.37\left(4 \mathrm{H}, \mathrm{m}, \mathrm{CH}_{2} \mathrm{NS}\right), 7.13\left(1 \mathrm{H}, \mathrm{d},{ }^{3} \mathrm{~J}=7.6 \mathrm{~Hz}\right.$, H6(Nf)), $7.45\left(1 \mathrm{H}, \mathrm{dd},{ }^{3} J=8.5 \mathrm{~Hz},{ }^{3} J=7.3 \mathrm{~Hz}, \mathrm{H} 3(\mathrm{Nf})\right), 7.50(1 \mathrm{H}$, dd, $\left.{ }^{3} J=8.7 \mathrm{~Hz},{ }^{3} J=7.6 \mathrm{~Hz}, \mathrm{H} 7(\mathrm{Nf})\right), 7.49\left(1 \mathrm{H}, \mathrm{dd},{ }^{3} J=7.3 \mathrm{~Hz}\right.$, $\left.{ }^{4} J=1.0 \mathrm{~Hz}, \mathrm{H} 2(\mathrm{Nf})\right), 8.43\left(1 \mathrm{H}, \mathrm{d},{ }^{3} J=8.7 \mathrm{~Hz}, \mathrm{H} 8(\mathrm{Nf})\right), 8.47(1 \mathrm{H}, \mathrm{d}$, $\left.{ }^{3} J=8.5 \mathrm{~Hz}, \mathrm{H} 4(\mathrm{Nf})\right) .{ }^{13} \mathrm{C} \mathrm{NMR}\left(\mathrm{CDCl}_{3}, 298 \mathrm{~K}\right) \delta_{\mathrm{c}} \mathrm{ppm}: 45.2(2 \mathrm{C}$, $\left.\mathrm{CH}_{3}\right), 49.2\left(2 \mathrm{C}, \mathrm{CH}_{2} \mathrm{NH}\right), 49.3\left(2 \mathrm{C}, \mathrm{CH}_{2} \mathrm{NH}\right), 53.7\left(2 \mathrm{C}, \mathrm{CH}_{2} \mathrm{NS}\right)$, $115.1(1 \mathrm{C}, \mathrm{CH}(\mathrm{Nf})), 119.6(1 \mathrm{C}, \mathrm{CH}(\mathrm{Nf})), 122.9(1 \mathrm{C}, \mathrm{CH}(\mathrm{Nf})), 127.9$ (1C, $\mathrm{CH}(\mathrm{Nf})), 128.0(1 \mathrm{C}, \mathrm{CH}(\mathrm{Nf})), 129.9$ (1C, $\mathrm{CH}(\mathrm{Nf})), 130.1$ (1C, $\mathrm{C}(\mathrm{Nf})), 130.2(1 \mathrm{C}, \mathrm{C}(\mathrm{Nf})), 135.5$ (1C, C(Nf)), 151.5 (1C, NC(Nf)).

5,5'-(1,4,7-Triazacyclononan-1,4-diylsulfonyl) bis ( N, Ndimethylnaphthalene-1-amine) (5). Obtained as the second product in the synthesis of compound 3. Eluent: $\mathrm{CHCl}_{3}-\mathrm{MeOH}$ 15:1, yellow viscous oil. Yield $172 \mathrm{mg}(29 \%) . \mathrm{m} / z$ (MALDI-TOF) found: 596.2319. $\mathrm{C}_{30} \mathrm{H}_{38} \mathrm{~N}_{5} \mathrm{O}_{4} \mathrm{~S}_{2}$ requires 596.2360 $[\mathrm{M}+\mathrm{H}]^{+}$. ${ }^{1} \mathrm{H}$ NMR $\left(\mathrm{CDCl}_{3}, 298 \mathrm{~K}\right) \delta_{\mathrm{H}}$ ppm: $1.83\left(1 \mathrm{H}\right.$, br. s, NH), $2.85\left(12 \mathrm{H}, \mathrm{s}, \mathrm{CH}_{3}\right)$, 3.19-3.24 (4H, m, CH $\mathrm{CHH}_{2}, 3.41-3.45$ (4H, m, $\left.\mathrm{CH}_{2} \mathrm{NS}\right), 3.67$ (4H, s, $\left.\mathrm{CH}_{2} \mathrm{NS}\right), 7.16\left(2 \mathrm{H}, \mathrm{d},{ }^{3} J=7.6 \mathrm{~Hz}, \mathrm{H} 6(\mathrm{Nf})\right), 7.48\left(2 \mathrm{H}, \mathrm{dd},{ }^{3} J=8.5 \mathrm{~Hz}\right.$, $\left.{ }^{3} J=7.5 \mathrm{~Hz}, \mathrm{H} 3(\mathrm{Nf})\right), 7.53\left(2 \mathrm{H}, \mathrm{dd},{ }^{3} J=8.7 \mathrm{~Hz},{ }^{3} J=7.6 \mathrm{~Hz}, \mathrm{H} 7(\mathrm{Nf})\right)$, $7.96\left(2 \mathrm{H}, \mathrm{dd},{ }^{3} J=7.5 \mathrm{~Hz},{ }^{4} J=1.0 \mathrm{~Hz}, \mathrm{H} 2(\mathrm{Nf})\right), 8.41\left(2 \mathrm{H}, \mathrm{d},{ }^{3} J=8.7 \mathrm{~Hz}\right.$, $\mathrm{H} 8(\mathrm{Nf})), 8.51\left(2 \mathrm{H}, \mathrm{d},{ }^{3} \mathrm{~J}=8.5 \mathrm{~Hz}, \mathrm{H} 4(\mathrm{Nf})\right) .{ }^{13} \mathrm{C} \mathrm{NMR}\left(\mathrm{CDCl}_{3}, 298\right.$ K) $\delta_{c} \mathrm{ppm}: 45.3\left(4 \mathrm{C}, \mathrm{CH}_{3}\right), 48.5\left(2 \mathrm{C}, \mathrm{CH}_{2} \mathrm{NH}\right), 52.7\left(2 \mathrm{C}, \mathrm{CH}_{2} \mathrm{NS}\right)$, $53.7\left(2 \mathrm{C}, \mathrm{CH}_{2} \mathrm{NS}\right), 115.3(2 \mathrm{C}, \mathrm{CH}(\mathrm{Nf})), 119.4(2 \mathrm{C}, \mathrm{CH}(\mathrm{Nf})), 120.0$ (2C, $\mathrm{CH}(\mathrm{Nf})), 128.2(4 \mathrm{C}, \mathrm{CH}(\mathrm{Nf})), 130.2$ (4C, C(Nf), $\mathrm{CH}(\mathrm{Nf}))$, 130.3 (2C, C(Nf)), 134.2 (2C, C(Nf)), 151.7 (2C, NC(Nf)).

5-((1,5,9-Triazacyclododecan-1-yl) sulfonyl)-N,N-dimethylnaphthalene-1-amine (4). Obtained from TACD (2) (342 mg, $2 \mathrm{mmol})$ and dansyl chloride (378 $\mathrm{mg}, 1.4 \mathrm{mmol})$ in the presence of potassium carbonate $(1380 \mathrm{mg}, 10 \mathrm{mmol})$ in $70 \mathrm{ml}$ acetonitrile. Eluent: $\mathrm{CH}_{2} \mathrm{Cl}_{2}-\mathrm{MeOH}$ 5:1, yellow viscous oil. Yield $166 \mathrm{mg}(28 \%) . \mathrm{m} / \mathrm{z}$ (MALDI-TOF) found: 405.2357. $\mathrm{C}_{21} \mathrm{H}_{33} \mathrm{~N}_{4} \mathrm{O}_{2} \mathrm{~S}$ requires 405.2324 $[\mathrm{M}+\mathrm{H}]^{+}$. ${ }^{1} \mathrm{H}$ NMR $\left(\mathrm{CDCl}_{3}, 298 \mathrm{~K}\right) \delta_{\mathrm{H}} \mathrm{ppm}: 1.83(2 \mathrm{H}$, quintet, $\left.{ }^{3} \mathrm{~J}=5.2 \mathrm{~Hz}, \mathrm{CCH}_{2} \mathrm{C}\right), 2.06\left(4 \mathrm{H}\right.$, quintet, $\left.{ }^{3} \mathrm{~J}=5.7 \mathrm{~Hz}, \mathrm{CCH}_{2} \mathrm{C}\right), 2.84$ $\left(6 \mathrm{H}, \mathrm{s}, \mathrm{CH}_{3}\right), 2.99\left(4 \mathrm{H}, \mathrm{t},{ }^{3} \mathrm{~J}=5.4 \mathrm{~Hz}, \mathrm{CH}_{2} \mathrm{~N}\right), 3.07\left(4 \mathrm{H}, \mathrm{t},{ }^{3} J=5.2 \mathrm{~Hz}\right.$, $\left.\mathrm{CH}_{2} \mathrm{~N}\right), 3.13\left(4 \mathrm{H}, \mathrm{t},{ }^{3} J=6.1 \mathrm{~Hz}, \mathrm{CH}_{2} \mathrm{~N}\right), 7.14\left(1 \mathrm{H}, \mathrm{d},{ }^{3} J=7.5 \mathrm{~Hz}\right.$, H6(Nf)), 7.47-7.53 (2H, m, H3, H7(Nf)), 8.07 (1H, d, ${ }^{3} J=7.5 \mathrm{~Hz}$, $\mathrm{H} 2(\mathrm{Nf})), 8.47\left(1 \mathrm{H}, \mathrm{d},{ }^{3} J=8.6 \mathrm{~Hz}, \mathrm{H} 8(\mathrm{Nf})\right), 8.55\left(1 \mathrm{H}, \mathrm{d},{ }^{3} J=8.5 \mathrm{~Hz}\right.$, $\mathrm{H} 4(\mathrm{Nf})$ ), two $\mathrm{NH}$ protons were not unambiguously assigned. ${ }^{13} \mathrm{C} \mathrm{NMR}\left(\mathrm{CDCl}_{3}, 298 \mathrm{~K}\right) \delta_{\mathrm{c}} \mathrm{ppm}: 22.1\left(1 \mathrm{C}, \mathrm{CCH}_{2} \mathrm{C}\right), 25.7(2 \mathrm{C}$, $\left.\mathrm{CCH}_{2} \mathrm{C}\right), 45.1\left(2 \mathrm{C}, \mathrm{CH}_{2} \mathrm{~N}\right), 45.3\left(2 \mathrm{C}, \mathrm{CH}_{3}\right), 49.1\left(2 \mathrm{C}, \mathrm{CH}_{2} \mathrm{~N}\right), 50.0$ $\left(2 \mathrm{C}, \mathrm{CH}_{2} \mathrm{~N}\right), 115.3(1 \mathrm{C}, \mathrm{CH}(\mathrm{Nf})), 119.0(1 \mathrm{C}, \mathrm{CH}(\mathrm{Nf})), 123.0(1 \mathrm{C}$, $\mathrm{CH}(\mathrm{Nf})), 128.2(1 \mathrm{C}, \mathrm{CH}(\mathrm{Nf})), 130.1$ (1C, C(Nf)), 130.7 (1C, CH(Nf)), 131.0 (2C, CH(Nf), C(Nf)), 131.2 (1C, C(Nf)), 151.7 (1C, NC(Nf)). 
5,5'-(1,5,9-Triazacyclododecane-1,5-diylsulfonyl)bis( $N, N$ dimethylnaphthalene-1-amine) (6). Obtained as the second product in the synthesis of compound 4. Eluent: $\mathrm{CH}_{2} \mathrm{Cl}_{2}-\mathrm{MeOH}$ 20:1, yellow crystalline powder, m.p. $158-160{ }^{\circ} \mathrm{C}$. Yield $288 \mathrm{mg}$ (65 \%). $\mathrm{m} / \mathrm{z}$ (MALDI-TOF) found: 638.2809. $\mathrm{C}_{33} \mathrm{H}_{44} \mathrm{~N}_{5} \mathrm{O}_{4} \mathrm{~S}_{2}$ requires $638.2835[\mathrm{M}+\mathrm{H}]^{+} .{ }^{1} \mathrm{H} \mathrm{NMR}\left(\mathrm{CDCl}_{3}, 298 \mathrm{~K}\right) \delta_{\mathrm{H}} \mathrm{ppm}: 1.67$ (4H, quintet, $\left.{ }^{3} J=5.5 \mathrm{~Hz}, \mathrm{CCH}_{2} \mathrm{C}\right), 1.87\left(4 \mathrm{H}\right.$, quintet, ${ }^{3} J=6.9 \mathrm{~Hz}$, $\left.\mathrm{CCH}_{2} \mathrm{C}\right), 2.61\left(2 \mathrm{H}, \mathrm{t},{ }^{3} \mathrm{~J}=5.4 \mathrm{~Hz}, \mathrm{CH}_{2} \mathrm{~N}\right), 2.87\left(12 \mathrm{H}, \mathrm{s}, \mathrm{CH}_{3}\right), 3.20$ $\left(4 \mathrm{H}, \mathrm{t},{ }^{3} \mathrm{~J}=6.9 \mathrm{~Hz}, \mathrm{CH}_{2} \mathrm{~N}\right), 3.27\left(4 \mathrm{H}, \mathrm{t},{ }^{3} \mathrm{~J}=5.9 \mathrm{~Hz}, \mathrm{CH}_{2} \mathrm{~N}\right), 7.16(2 \mathrm{H}$, d, $\left.{ }^{3} J=7.5 \mathrm{~Hz}, \mathrm{H} 6(\mathrm{Nf})\right), 7.47-7.53$ (4H, m, H3, H7(Nf)), 8.14 (2H, dd, $\left.{ }^{3} J=7.3 \mathrm{~Hz},{ }^{4} J=0.8 \mathrm{~Hz}, \mathrm{H} 2(\mathrm{Nf})\right), 8.35\left(2 \mathrm{H}, \mathrm{d},{ }^{3} J=8.7 \mathrm{~Hz}, \mathrm{H} 8(\mathrm{Nf})\right)$, $8.52\left(2 \mathrm{H}, \mathrm{d},{ }^{3} J=8.5 \mathrm{~Hz}, \mathrm{H} 4(\mathrm{Nf})\right)$, $\mathrm{NH}$ proton was not assigned. ${ }^{13} \mathrm{C}$ NMR $\left(\mathrm{CDCl}_{3}, 298 \mathrm{~K}\right) \delta_{\mathrm{c}} \mathrm{ppm}: 23.8\left(1 \mathrm{C}, \mathrm{CCH}_{2} \mathrm{C}\right), 27.8(2 \mathrm{C}$, $\left.\mathrm{CCH}_{2} \mathrm{C}\right), 42.9\left(2 \mathrm{C}, \mathrm{CH}_{2} \mathrm{~N}\right), 45.4\left(6 \mathrm{C}, \mathrm{CH}_{3}, \mathrm{CH}_{2} \mathrm{~N}\right), 45.8\left(2 \mathrm{C}, \mathrm{CH}_{2} \mathrm{~N}\right)$, $115.1(2 \mathrm{C}, \mathrm{CH}(\mathrm{Nf})), 119.6(2 \mathrm{C}, \mathrm{CH}(\mathrm{Nf})), 123.1$ (2C, CH(Nf)), 127.9 (2C, $\mathrm{CH}(\mathrm{Nf})), 130.0(2 \mathrm{C}, \mathrm{C}(\mathrm{Nf})), 130.1(2 \mathrm{C}, \mathrm{CH}(\mathrm{Nf})), 130.3$ (4C, $\mathrm{CH}(\mathrm{Nf}), \mathrm{C}(\mathrm{Nf})), 134.0(2 \mathrm{C}, \mathrm{C}(\mathrm{Nf})), 135.6(2 \mathrm{C}, \mathrm{NC}(\mathrm{Nf}))$.

Method for the synthesis of dansyl and bromobenzyl derivatives of triazacycloalkanes 7-9. A one-neck flask equipped with a magnetic stirrer was charged with a corresponding dansyl derivative of TACN (3) or TACD (4) which was solubilized in $0.3-0.5 \mathrm{ml}$ dichloromethane, then acetonitrile $(2-8 \mathrm{ml})$ was added to make a solution, followed by potassium carbonate, then appropriate bromobenzyl bromide was added in one portion. The reaction mixture was stirred at ambient temperature for $24 \mathrm{~h}$, then the residue was filtered off, washed with dichloromethane $(5 \mathrm{ml})$, the combined organic fractions were evaporated in vacuo and, if necessary, chromatographed on silica gel using a sequence of eluents: $\mathrm{CH}_{2} \mathrm{Cl}_{2}, \mathrm{CH}_{2} \mathrm{Cl}_{2}-\mathrm{MeOH}$ 100:1-2:1. In the case when chromatographic purification was unnecessary, the reaction mixture was dissolved in $5 \mathrm{ml}$ dichloromethane, washed with water, dried over anhydrous sodium sulfate and evaporated in vacuo to dryness

5-(4,7-Bis(3-bromobenzyl)-1,4,7-triazacyclononan1 -ylsulfonyl)-N,N-dimethylnaphthalene-1-amine (7). Obtained from compound 3 (405 mg, $1.12 \mathrm{mmol}$ ), 3-bromobenzyl bromide $(500 \mathrm{mg}, 2 \mathrm{mmol})$ in the presence of potassium carbonate $(770 \mathrm{mg}, 5.58 \mathrm{mmol})$ in $6 \mathrm{ml}$ acetonitrile. Yellow glassy solid. Yield $577 \mathrm{mg}$ (83 \%). m/z (MALDI-TOF) found: 699.1034 . $\mathrm{C}_{32} \mathrm{H}_{37} \mathrm{Br}_{2} \mathrm{~N}_{4} \mathrm{O}_{2} \mathrm{~S}$ requires $699.1004[\mathrm{M}+\mathrm{H}]^{+}$. ${ }^{1} \mathrm{H} \mathrm{NMR}\left(\mathrm{CDCl}_{3}\right.$, $298 \mathrm{~K}) \delta_{\mathrm{H}}$ ppm: $2.68\left(4 \mathrm{H}\right.$, br. s, $\left.\mathrm{CH}_{2} \mathrm{~N}\right), 2.84\left(6 \mathrm{H}, \mathrm{s}, \mathrm{CH}_{3}\right), 3.04$ $\left(4 \mathrm{H}\right.$, br. s, $\left.\mathrm{CH}_{2} \mathrm{~N}\right), 3.45$ (4H, br. s, $\left.\mathrm{CH}_{2} \mathrm{NS}\right), 3.60\left(4 \mathrm{H}, \mathrm{s}, \mathrm{PhCH}_{2} \mathrm{~N}\right)$, 7.13-7.18 (3H, m, H6(Nf), H5(Ph)), 7.23 (2H, br. s, H6(Ph)), 7.35 $\left(2 \mathrm{H}, \mathrm{d},{ }^{3} J=7.8 \mathrm{~Hz}, \mathrm{H} 4(\mathrm{Ph})\right), 7.44(2 \mathrm{H}$, br. s, H2(Ph)), $7.47(1 \mathrm{H}, \mathrm{dd}$, $\left.{ }^{3} J=8.5 \mathrm{~Hz},{ }^{3} J=7.5 \mathrm{~Hz}, \mathrm{H} 3(\mathrm{Nf})\right), 7.53\left(1 \mathrm{H}, \mathrm{dd},{ }^{3} J=8.7 \mathrm{~Hz},{ }^{3} J=7.7 \mathrm{~Hz}\right.$, $\mathrm{H} 7(\mathrm{Nf})), 8.01\left(1 \mathrm{H}, \mathrm{dd},{ }^{3} J=7.5 \mathrm{~Hz},{ }^{4} J=0.9 \mathrm{~Hz}, \mathrm{H} 2(\mathrm{Nf})\right), 8.43(1 \mathrm{H}$, $\left.\mathrm{d},{ }^{3} J=8.7 \mathrm{~Hz}, \mathrm{H} 8(\mathrm{Nf})\right), 8.49\left(1 \mathrm{H}, \mathrm{d},{ }^{3} J=8.5 \mathrm{~Hz}, \mathrm{H} 4(\mathrm{Nf})\right) .{ }^{13} \mathrm{C}$ NMR $\left(\mathrm{CDCl}_{3}, 298 \mathrm{~K}\right) \delta_{c} \mathrm{ppm}: 45.1\left(2 \mathrm{C}, \mathrm{CH}_{3}\right), 50.2\left(2 \mathrm{C}, \mathrm{CH}_{2} \mathrm{~N}\right), 54.9(2 \mathrm{C}$, $\left.\mathrm{CH}_{2} \mathrm{~N}\right), 55.1\left(2 \mathrm{C}, \mathrm{CH}_{2} \mathrm{~N}\right), 61.4\left(2 \mathrm{C}, \mathrm{NCH}_{2} \mathrm{Ph}\right), 115.0(1 \mathrm{C}, \mathrm{CH}(\mathrm{Nf}))$, $119.5(1 \mathrm{C}, \mathrm{CH}(\mathrm{Nf})), 122.1(2 \mathrm{C}, \mathrm{C} 3(\mathrm{Ph})), 122.9(1 \mathrm{C}, \mathrm{CH}(\mathrm{Nf})), 127.4$ (2C, $\mathrm{CH}(\mathrm{Ph})), 127.8(1 \mathrm{C}, \mathrm{CH}(\mathrm{Nf})), 128.2(1 \mathrm{C}, \mathrm{CH}(\mathrm{Nf})), 129.7$ (2C, $\mathrm{CH}(\mathrm{Ph})), 129.8(1 \mathrm{C}, \mathrm{CH}(\mathrm{Nf})), 130.0(2 \mathrm{CH}(\mathrm{Ph}), 2 \mathrm{C}(\mathrm{Nf})), 131.6(2 \mathrm{C}$, $\mathrm{CH}(\mathrm{Ph})), 135.0$ (1C, C(Nf)), 142.0 (2C, C1(Ph)), 151.4 (1C, C(Nf)).

5-(4,7-Bis(4-bromobenzyl)-1,4,7-triazacyclononan-1-ylsulfonyl)- $N, N$-dimethylnaphthalene-1-amine (8). Obtained from compound 3 (340 $\mathrm{mg}, 0.94 \mathrm{mmol})$, 4-bromobenzyl bromide $(350 \mathrm{mg}, 1.5 \mathrm{mmol})$, in the presence of potassium carbonate $(400 \mathrm{mg}, 2.90 \mathrm{mmol})$ in $8 \mathrm{ml}$ acetonitrile. Yellow glassy solid, yield $520 \mathrm{mg}$ (99\%). m/z (MALDI-TOF) found: 699.1045 . $\mathrm{C}_{32} \mathrm{H}_{37} \mathrm{Br}_{2} \mathrm{~N}_{4} \mathrm{O}_{2} \mathrm{~S}$ requires $699.1004[\mathrm{M}+\mathrm{H}]^{+} .{ }^{1} \mathrm{H}$ NMR $\left(\mathrm{CDCl}_{3}, 298\right.$ K) $\delta_{\mathrm{H}}$ ppm: $2.63\left(4 \mathrm{H}\right.$, br. s, $\left.\mathrm{CH}_{2} \mathrm{~N}\right), 2.84\left(6 \mathrm{H}, \mathrm{s}, \mathrm{CH}_{3}\right) 3.02(4 \mathrm{H}$, br. s, $\left.\mathrm{CH}_{2} \mathrm{~N}\right), 3.44$ (4H, br. s, $\left.\mathrm{CH}_{2} \mathrm{NS}\right), 3.57\left(2 \mathrm{H}, \mathrm{s}, \mathrm{PhCH}_{2} \mathrm{~N}\right), 7.15(4 \mathrm{H}, \mathrm{d}$, ${ }^{3} J=8.3 \mathrm{~Hz}, \mathrm{H} 2, \mathrm{H} 2$ ' $\left.(\mathrm{Ph})\right), 7.24\left(1 \mathrm{H}, \mathrm{d},{ }^{3} J=7.6 \mathrm{~Hz}, \mathrm{H} 6(\mathrm{Nf})\right), 7.39(4 \mathrm{H}$, d, ${ }^{3} J=8.3 \mathrm{~Hz}, \mathrm{H} 3, \mathrm{H} 3$ ' $\left.(\mathrm{Ph})\right), 7.46\left(1 \mathrm{H}, \mathrm{dd},{ }^{3} J=8.5 \mathrm{~Hz},{ }^{3} J=7.3 \mathrm{~Hz}\right.$, $\mathrm{H} 3(\mathrm{Nf})), 7.51\left(1 \mathrm{H}, \mathrm{dd},{ }^{3} J=8.6 \mathrm{~Hz},{ }^{3} J=7.6 \mathrm{~Hz}, \mathrm{H} 7(\mathrm{Nf})\right), 7.99(1 \mathrm{H}$, d, $\left.{ }^{3} J=7.3 \mathrm{~Hz}, \mathrm{H} 2(\mathrm{Nf})\right), 8.40\left(1 \mathrm{H}, \mathrm{d},{ }^{3} J=8.6 \mathrm{~Hz}, \mathrm{H} 8(\mathrm{Nf})\right), 8.49(1 \mathrm{H}$, $\left.\mathrm{d},{ }^{3} J=8.5 \mathrm{~Hz}, \mathrm{H} 4(\mathrm{Nf})\right) .{ }^{13} \mathrm{C} \mathrm{NMR}\left(\mathrm{CDCl}_{3}, 298 \mathrm{~K}\right) \delta_{\mathrm{c}} \mathrm{ppm:} 45.3$ $\left(2 \mathrm{C}, \mathrm{CH}_{3}\right), 50.2\left(2 \mathrm{C}, \mathrm{CH}_{2} \mathrm{~N}\right), 55.2\left(2 \mathrm{C}, \mathrm{CH}_{2} \mathrm{~N}\right), 55.4\left(2 \mathrm{C}, \mathrm{CH}_{2} \mathrm{~N}\right)$, $61.7\left(2 \mathrm{C}, \mathrm{PhCH}_{2} \mathrm{~N}\right), 115.2(1 \mathrm{C}, \mathrm{CH}(\mathrm{Nf})), 119.6(1 \mathrm{C}, \mathrm{CH}(\mathrm{Nf})), 120.7$ (2C, $\mathrm{C} 4(\mathrm{Ph})), 123.0(1 \mathrm{C}, \mathrm{CH}(\mathrm{Nf})), 128.0$ (1C, CH(Nf)), $128.4(1 \mathrm{C}$, $\mathrm{CH}(\mathrm{Nf})), 129.9$ (1C, C(Nf)), 130.2 (1C, C(Nf)), 130.7 (4CH(Ph), $\mathrm{CH}(\mathrm{Nf})), 131.3$ (4C, $\mathrm{CH}(\mathrm{Ph})), 135.1$ (1C, C(Nf)), 138.7 (2C, $\mathrm{Cl}(\mathrm{Ph})$ ), $151.6(1 \mathrm{C}, \mathrm{NC}(\mathrm{Nf}))$.

5-((5,9-Bis(3-bromobenzyl)-1,5,9-triazacyclododecan-1-yl)sulfonyl)-N,N-dimethylnaphthalene-1-amine (9). Obtained from compound 4 (166 mg, $0.41 \mathrm{mmol}$ ), 3-bromobenzyl bromide (200 $\mathrm{mg}, 0.8 \mathrm{mmol}$ ), in the presence of potassium carbonate (500 mg, $3.62 \mathrm{mmol}$ ) in $4 \mathrm{ml}$ acetonitrile. Eluent: $\mathrm{CH}_{2} \mathrm{Cl}_{2}-\mathrm{MeOH}$ 100:1, yellow glassy solid. Yield $113 \mathrm{mg}(38 \%) . \mathrm{m} / z$ (MALDI-TOF) found: 741.1449. $\mathrm{C}_{35} \mathrm{H}_{43} \mathrm{Br}_{2} \mathrm{~N}_{4} \mathrm{O}_{2} \mathrm{~S}$ requires $741.1473[\mathrm{M}+\mathrm{H}]^{+}$. ${ }^{1} \mathrm{H}$ NMR $\left(\mathrm{CDCl}_{3}, 298 \mathrm{~K}\right) \delta_{\mathrm{H}}$ ppm: $1.57\left(4 \mathrm{H}\right.$, br. s, $\left.\mathrm{CCH}_{2} \mathrm{C}\right)$, $1.67\left(2 \mathrm{H}\right.$, br. quintet, $\left.{ }^{3} \mathrm{~J}_{\mathrm{obs}}=5.1 \mathrm{~Hz}, \mathrm{CCH}_{2} \mathrm{C}\right), 2.22(4 \mathrm{H}$, br. $\mathrm{t}$, $\left.{ }^{3} \mathrm{~J}_{\mathrm{obs}}=4.9 \mathrm{~Hz}, \mathrm{CH}_{2} \mathrm{~N}\right), 2.58\left(4 \mathrm{H}\right.$, br.s, $\left.\mathrm{CH}_{2} \mathrm{~N}\right), 2.86\left(6 \mathrm{H}, \mathrm{s}, \mathrm{CH}_{3}\right)$, $3.57\left(4 \mathrm{H}, \mathrm{t},{ }^{3} \mathrm{~J}=7.6 \mathrm{~Hz}, \mathrm{CH}_{2} \mathrm{~N}\right), 3.39\left(4 \mathrm{H}, \mathrm{s}, \mathrm{PhCH}_{2} \mathrm{~N}\right), 7.14-7.18$ $(5 \mathrm{H}, \mathrm{m}, \mathrm{H6}(\mathrm{Nf}), \mathrm{H}(\mathrm{Ph})), 7.34-7.38(4 \mathrm{H}, \mathrm{m}, \mathrm{H}(\mathrm{Ph})), 7.46(1 \mathrm{H}, \mathrm{dd}$, $\left.{ }^{3} J=8.5 \mathrm{~Hz},{ }^{3} J=7.3 \mathrm{~Hz}, \mathrm{H} 3(\mathrm{Nf})\right), 7.51\left(1 \mathrm{H}, \mathrm{dd},{ }^{3} J=8.7 \mathrm{~Hz},{ }^{3} J=7.6 \mathrm{~Hz}\right.$, $\mathrm{H} 7(\mathrm{Nf})), 8.13\left(1 \mathrm{H}, \mathrm{dd},{ }^{3} J=7.3 \mathrm{~Hz},{ }^{4} J=0.9 \mathrm{~Hz}, \mathrm{H} 2(\mathrm{Nf})\right), 8.18(1 \mathrm{H}, \mathrm{d}$, $\left.{ }^{3} J=8.7 \mathrm{~Hz}, \mathrm{H} 7(\mathrm{Nf})\right), 8.48\left(1 \mathrm{H}, \mathrm{d},{ }^{3} J=8.5 \mathrm{~Hz}, \mathrm{H} 4(\mathrm{Nf})\right) .{ }^{13} \mathrm{C} \mathrm{NMR}$ $\left(\mathrm{CDCl}_{3}, 298 \mathrm{~K}\right) \delta_{\mathrm{c}}$ ppm: $23.1\left(2 \mathrm{C}, \mathrm{CCH}_{2} \mathrm{C}\right), 23.9\left(1 \mathrm{C}, \mathrm{CCH}_{2} \mathrm{C}\right), 41.3$ $\left(2 \mathrm{C}, \mathrm{CH}_{2} \mathrm{~N}\right), 45.4\left(2 \mathrm{C}, \mathrm{CH}_{3}\right), 48.3\left(2 \mathrm{C}_{2} \mathrm{CH}_{2} \mathrm{~N}\right), 51.0\left(2 \mathrm{C}, \mathrm{CH}_{2} \mathrm{~N}\right)$, $57.7\left(2 \mathrm{C}, \mathrm{PhCH}_{2} \mathrm{~N}\right), 115.0$ (1C, CH(Nf)), 119.5 (1C, CH(Nf)), 122.3 (2C, $\mathrm{C} 3(\mathrm{Ph})), 123.1(1 \mathrm{C}, \mathrm{CH}(\mathrm{Nf})), 127.4(2 \mathrm{C}, \mathrm{CH}(\mathrm{Ph})), 127.8$ (1C, $\mathrm{CH}(\mathrm{Nf})), 129.5$ (1C, CH(Nf)), 129.8 (2C, $\mathrm{CH}(\mathrm{Ph})), 129.9$ (br.s, $\mathrm{CH}(\mathrm{Nf}), 2 \mathrm{CH}(\mathrm{Ph}), 2 \mathrm{C}(\mathrm{Nf})), 131.8(2 \mathrm{C}, \mathrm{CH}(\mathrm{Ph})), 135.7$ (1C, $\mathrm{C}(\mathrm{Nf}))$, $141.8(2 \mathrm{C}, \mathrm{C} 1(\mathrm{Ph})), 151.6(1 \mathrm{C}, \mathrm{NC}(\mathrm{Nf}))$.

Method for the synthesis of the cryptands 11-13. A twoneck flask equipped with a magnetic stirrer and reflux condenser, flushed with dry argon, was charged with corresponding triazacycloalkane derivative 7-9, $\mathrm{Pd}(\mathrm{dba})_{2}$ (16 mol\%), DavePhos (18 mol\%), absolute dioxane. The mixture was stirred for 2-3 min, then trioxadiamine $\mathbf{1 0}$ was added followed by $t \mathrm{BuONa}$. The reaction mixture was stirred at reflux for $24 \mathrm{~h}$, cooled down to ambient temperature, the residue was filtered off, washed with dichloromethane $(5 \mathrm{ml})$, combined organic fractions were evaporated in vacuo, and the residue was chromatographed on silica gel using a sequence of eluents $\mathrm{CH}_{2} \mathrm{Cl}_{2}, \mathrm{CH}_{2} \mathrm{Cl}_{2}-\mathrm{MeOH}$ 100:1-2:1, $\mathrm{CH}_{2} \mathrm{Cl}_{2}-$ $\mathrm{MeOH}-\mathrm{NH}_{3}$ 100:20:1-10:4:1.

5-(10,13,16-Trioxa-6,20-diaza-3(1,4)-triazacyclononane1,5(1,3)-dibenzenocycloicosaphan-37-ylsulfonyl)- $N, N$-dimethylnaphthalenyl-1-amine (11). Obtained from compound 7 (140 mg, $0.2 \mathrm{mmol})$, trioxadiamine $10(44 \mathrm{mg}, 0.2 \mathrm{mmol})$, in the presence of Pd(dba) (18 mg, $0.032 \mathrm{mmol})$, DavePhos (14 mg, $0.036 \mathrm{mmol})$, $t \mathrm{BuONa}(58 \mathrm{mg}, 0.6 \mathrm{mmol})$ in $10 \mathrm{ml}$ dioxane. Eluent: $\mathrm{CH}_{2} \mathrm{Cl}_{2}$ $\mathrm{MeOH} 3: 1$, yield $37 \mathrm{mg}$ (24\%), yellow glassy solid. UV-Vis $\left(\mathrm{CH}_{3} \mathrm{CN}\right) \lambda_{\max }(\log \varepsilon) \mathrm{nm}: 305$ (3.76), 340 (3.48). m/z (MALDITOF) found: $759.4223 . \mathrm{C}_{42} \mathrm{H}_{59} \mathrm{~N}_{6} \mathrm{O}_{5} \mathrm{~S}$ requires $759.4268[\mathrm{M}+\mathrm{H}]^{+} .{ }^{1} \mathrm{H}$ NMR $\left(\mathrm{CDCl}_{3}, 298 \mathrm{~K}\right) \delta_{\mathrm{H}}$ ppm: $1.85\left(4 \mathrm{H}\right.$, br. quintet, ${ }^{3}{ }_{\text {obs }}=5.7 \mathrm{~Hz}$, $\left.\mathrm{CH}_{2} \mathrm{CH}_{2} \mathrm{CH}_{2}\right), 2.86\left(4 \mathrm{H}\right.$, br. s, $\left.\mathrm{CH}_{2} \mathrm{~N}\right), 2.87\left(6 \mathrm{H}, \mathrm{s}, \mathrm{CH}_{3}\right) 3.08-4.00$ $\left(12 \mathrm{H}\right.$, br. $\left.\mathrm{m}, \mathrm{CH}_{2} \mathrm{~N}, \mathrm{PhCH}_{2} \mathrm{~N}\right), 3.24\left(4 \mathrm{H}\right.$, br. t, ${ }^{3}{ }^{2}=4.8 \mathrm{~Hz}$, $\left.\mathrm{CH}_{2} \mathrm{NPh}\right), 3.55-3.60\left(8 \mathrm{H}\right.$, br. $\left.\mathrm{m}, \mathrm{CH}_{2} \mathrm{O}\right), 3.61-3.66(4 \mathrm{H}$, br. m, $\left.\mathrm{CH}_{2} \mathrm{O}\right), 6.58(2 \mathrm{H}$, br. s, $\mathrm{H}(\mathrm{Ph})), 6.60\left(2 \mathrm{H}\right.$, br. d $\left.,{ }^{3} \mathrm{~J}_{o b s}=6.9 \mathrm{~Hz}, \mathrm{H}(\mathrm{Ph})\right)$, $6.84(2 \mathrm{H}$, br. s, $\mathrm{H} 2(\mathrm{Ph})), 7.10\left(2 \mathrm{H}, \mathrm{t},{ }^{3} J=7.7 \mathrm{~Hz}, \mathrm{H} 5(\mathrm{Ph})\right), 7.15(1 \mathrm{H}$, d, $\left.{ }^{3} J=7.6 \mathrm{~Hz}, \mathrm{H6}(\mathrm{Nf})\right), 7.49\left(1 \mathrm{H}, \mathrm{t},{ }^{3} J_{o b s}=7.8 \mathrm{~Hz}, \mathrm{H} 3(\mathrm{Nf})\right), 7.53(1 \mathrm{H}$, t, $\left.{ }^{3} J_{o b s}=8.2 \mathrm{~Hz}, \mathrm{H} 7(\mathrm{Nf})\right), 7.98(1 \mathrm{H}$, br. s, H2(Nf)), $8.34(1 \mathrm{H}$, br. d, $\left.{ }^{3} J=7.6 \mathrm{~Hz}, \mathrm{H} 8(\mathrm{Nf})\right), 8.54$ (1H, br. d, $\left.{ }^{3} J=8.2 \mathrm{~Hz}, \mathrm{H} 4(\mathrm{Nf})\right)$, two $\mathrm{NH}$ protons were not unambiguously assigned. ${ }^{13} \mathrm{C} \mathrm{NMR}\left(\mathrm{CDCl}_{3}\right.$, $298 \mathrm{~K}) \delta_{c}$ ppm: $28.7\left(2 \mathrm{C}, \mathrm{CCH}_{2} \mathrm{C}\right), 41.4\left(2 \mathrm{C}, \mathrm{CH}_{2} \mathrm{NPh}\right), 45.3(2 \mathrm{C}$, $\left.\mathrm{CH}_{3}\right), 47.0-57.0\left(6 \mathrm{C}\right.$, br. m, $\left.\mathrm{CH}_{2} \mathrm{~N}\right), 61.7\left(2 \mathrm{C}\right.$, br. $\mathrm{s}, \Delta v_{1 / 2}=100 \mathrm{~Hz}$, $\left.\mathrm{PhCH}_{2} \mathrm{~N}\right), 69.4\left(2 \mathrm{C}, \mathrm{CH}_{2} \mathrm{O}\right), 70.1\left(2 \mathrm{C}, \mathrm{CH}_{2} \mathrm{O}\right), 70.5\left(2 \mathrm{C}, \mathrm{CH}_{2} \mathrm{O}\right)$, $112.7\left(2 \mathrm{C}\right.$, br. s, $\left.\Delta v_{1 / 2}=60 \mathrm{~Hz}, \mathrm{CH}(\mathrm{Ph})\right), 114.3^{2}\left(2 \mathrm{C}\right.$, br. s, $\Delta v_{1 / 2}=30 \mathrm{~Hz}$, $(\mathrm{CH}(\mathrm{Ph})), 115.4(1 \mathrm{C}, \mathrm{CH}(\mathrm{Nf})), 118.1(2 \mathrm{C}, \mathrm{CH}(\mathrm{Ph})), 119.0$ (1C, br. s, $\left.\Delta v_{1 / 2}=30 \mathrm{~Hz}, \mathrm{CH}(\mathrm{Nf})\right), 123.1(1 \mathrm{C},(\mathrm{CH}(\mathrm{Nf})), 128.2-131.0$ (br. m, $3 \mathrm{CH}(\mathrm{Nf}), 2 \mathrm{C} 5(\mathrm{Ph}), 2 \mathrm{C}(\mathrm{Nf})), 134.4(1 \mathrm{C}, \mathrm{C}(\mathrm{Nf})), 137.5(2 \mathrm{C}, \mathrm{Cl}(\mathrm{Ph}))$, 149.2 (2C, br. s, $\left.\Delta v_{1 / 2}=20 \mathrm{~Hz}, \mathrm{C} 3(\mathrm{Ph})\right), 151.9$ (1C, NC(Nf)). 
5-(10,13,16-Trioxa-6,20-diaza-3(1,4)-triazacyclononan$1,5(1,4)$-dibenzenocycloicosaphan- $3^{7}-y l$ sulfonyl) $-N, N$ dimethylnaphthalene-1-amine (12). Obtained from compound 8 (125 mg, $0.178 \mathrm{mmol})$, trioxadiamine 10 (39 mg, $0.178 \mathrm{mmol}$ ), in the presence of $\mathrm{Pd}(\mathrm{dba})_{2}(16 \mathrm{mg}, 0.028 \mathrm{mmol})$, DavePhos (13 mg, $0.032 \mathrm{mmol}), t$ BuONa $(51 \mathrm{mg}, 0.53 \mathrm{mmol})$, in $10 \mathrm{ml}$ dioxane. Eluent: $\mathrm{CH}_{2} \mathrm{Cl}_{2}-\mathrm{MeOH} 2: 1$, yield $21 \mathrm{mg}$ (15\%), yellow glassy solid. UV-Vis $\left(\mathrm{CH}_{3} \mathrm{CN}\right) \lambda_{\max }(\lg \varepsilon) \mathrm{nm}: 305$ (3.56), 340 (3.48). $\mathrm{m} / z$ (MALDI-TOF) found: $759.4223 . \mathrm{C}_{42} \mathrm{H}_{59} \mathrm{~N}_{6} \mathrm{O}_{5} \mathrm{~S}$ requires $759.4268[\mathrm{M}+\mathrm{H}]^{+} .{ }^{1} \mathrm{H} \mathrm{NMR}\left(\mathrm{CDCl}_{3}, 298 \mathrm{~K}\right) \delta_{\mathrm{H}} \mathrm{ppm}: 1.88(4 \mathrm{H}, \mathrm{br}$. quintet, $\left.{ }^{3} \mathrm{~J}_{\text {obs }}=5.7 \mathrm{~Hz}, \mathrm{CCH}_{2} \mathrm{C}\right), 2.88\left(6 \mathrm{H}, \mathrm{s}, \mathrm{CH}_{3}\right), 3.10-4.00(16 \mathrm{H}$, br. m, $\left.\mathrm{CH}_{2} \mathrm{~N}, \mathrm{PhCH}_{2} \mathrm{~N}\right), 3.26\left(4 \mathrm{H}\right.$, br. t, ${ }^{3} \mathrm{~J}_{o b s}=5.8 \mathrm{~Hz}, \mathrm{CH}_{2} \mathrm{NPh}$ ), $3.58-3.63\left(8 \mathrm{H}, \mathrm{m}, \mathrm{CH}_{2} \mathrm{O}\right), 3.67-3.71\left(4 \mathrm{H}, \mathrm{obs}, \mathrm{CH}_{2} \mathrm{O}\right), 4.52(2 \mathrm{H}$, br. s, NH), $6.55\left(4 \mathrm{H}, \mathrm{d},{ }^{3} J_{o b s}=8.1 \mathrm{~Hz}, \mathrm{H} 2, \mathrm{H} 2\right.$ ' $\left.(\mathrm{Ph})\right), 7.03(4 \mathrm{H}$, br. s, H3, H3'(Ph)), $7.19\left(1 \mathrm{H}, \mathrm{d}^{3}{ }^{3} J_{o b s}=7.3 \mathrm{~Hz}, \mathrm{H} 6(\mathrm{Nf})\right), 7.52(1 \mathrm{H}$, $\left.\mathrm{t},{ }^{3} J_{o b s}=8.0 \mathrm{~Hz}, \mathrm{H} 3(\mathrm{Nf})\right), 7.55\left(1 \mathrm{H}, \mathrm{t},{ }^{3} J_{o b s}=7.9 \mathrm{~Hz}, \mathrm{H} 7(\mathrm{Nf})\right), 8.04$ $\left(1 \mathrm{H}\right.$, br. s, H2(Nf)), $8.31\left(1 \mathrm{H}, \mathrm{d},{ }^{3} J_{\text {obs }}=8.8 \mathrm{~Hz}, \mathrm{H} 8(\mathrm{Nf})\right), 8.56(1 \mathrm{H}$, $\left.\mathrm{d},{ }^{3} J=8.6 \mathrm{~Hz}, \mathrm{H} 4(\mathrm{Nf})\right) .{ }^{13} \mathrm{C} \mathrm{NMR}\left(\mathrm{CDCl}_{3}, 298 \mathrm{~K}\right) \delta \mathrm{ppm}: 28.7$ $\left(2 \mathrm{C}, \mathrm{CCH}_{2} \mathrm{C}\right), 42.1\left(\mathrm{CH}_{2} \mathrm{NHPh}\right), 45.4\left(2 \mathrm{C}, \mathrm{CH}_{3}\right), 47.9-53.5(6 \mathrm{C}$, br. m, $\left.\mathrm{CH}_{2} \mathrm{~N}\right), 60.6\left(2 \mathrm{C}\right.$, br. s, $\left.\Delta v_{1 / 2}=50 \mathrm{~Hz}, \mathrm{PhCH}_{2} \mathrm{~N}\right), 70.1(2 \mathrm{C}$, $\left.\mathrm{CH}_{2} \mathrm{O}\right), 70.2\left(2 \mathrm{C}, \mathrm{CH}_{2} \mathrm{O}\right), 70.7\left(2 \mathrm{C}, \mathrm{CH}_{2} \mathrm{O}\right), 112.9(4 \mathrm{C}, \mathrm{CH}(\mathrm{Ph}))$, $115.5(1 \mathrm{C}, \mathrm{CH}(\mathrm{Nf})), 118.7(1 \mathrm{C}, \mathrm{CH}(\mathrm{Nf})), 123.1(1 \mathrm{C}, \mathrm{CH}(\mathrm{Nf})), 128.6$ $(1 \mathrm{C}, \mathrm{CH}(\mathrm{Nf})), 130.1(1 \mathrm{C}, \mathrm{C}(\mathrm{Nf})), 130.3(2 \mathrm{C}, \mathrm{Cl}(\mathrm{Ph})), 130.7(1 \mathrm{C}$, $\mathrm{C}(\mathrm{Nf})), 131.1(1 \mathrm{C}, \mathrm{C}(\mathrm{Nf})), 131.6(6 \mathrm{C}, 4 \mathrm{CH}(\mathrm{Ph}), 2 \mathrm{CH}(\mathrm{Nf})), 149.0$ $(2 \mathrm{C}, \mathrm{C} 4(\mathrm{Ph})), 152.0(1 \mathrm{C}, \mathrm{NC}(\mathrm{Nf}))$.

5-(10,13,16-Trioxa- $3^{1}, 3^{5}, 3^{9}, 6,20$-pentaaza-3(1,5)-cyclododecana-1,5(1,3)-dibenzenocycloicosaphan- $3^{9}-y l$ lsulfonyl) $-N, N$ dimethylnaphthalene-1-amine (13). Obtained from compound 9 (113 mg, $0.152 \mathrm{mmol}$ ), trioxadiamine 10 (33 mg, $0.152 \mathrm{mmol}$ ), in the presence of Pd(dba) ${ }_{2}(14 \mathrm{mg}, 0.0243 \mathrm{mmol})$, DavePhos (11 mg, $0.0274 \mathrm{mmol}), t \mathrm{BuONa}(58 \mathrm{mg}, 0.608 \mathrm{mmol})$, in $10 \mathrm{ml}$ dioxane. Eluent: $\mathrm{CH}_{2} \mathrm{Cl}_{2}-\mathrm{MeOH} 10: 1$, yield $32 \mathrm{mg}$ (26\%), yellow glassy solid. UV-Vis $\left(\mathrm{CH}_{3} \mathrm{CN}\right) \lambda_{\max }(\log \varepsilon) \mathrm{nm}: 305$ (3.59), 340 (3.43). $\mathrm{m} / \mathrm{z}$ (MALDI-TOF) found: 801.4761. $\mathrm{C}_{45} \mathrm{H}_{65} \mathrm{~N}_{6} \mathrm{O}_{5} \mathrm{~S}$ requires 801.4737 $[\mathrm{M}+\mathrm{H}]^{+} .{ }^{1} \mathrm{H}$ NMR $\left(\mathrm{CDCl}_{3}, 298 \mathrm{~K}\right) \delta_{\mathrm{H}} \mathrm{ppm}: 1.50-2.70(14 \mathrm{H}$, br. m, $\mathrm{NCCH}_{2} \mathrm{CN}, \mathrm{CH}_{2} \mathrm{~N}$ ), 1.87 (4H, quintet, ${ }^{3} J=6.3 \mathrm{~Hz}, \mathrm{NCCH}_{2} \mathrm{CO}$ ), $2.88\left(6 \mathrm{H}, \mathrm{s}, \mathrm{CH}_{3}\right) 3.10-3.70\left(2 \mathrm{H}\right.$, br. m, $\left.\mathrm{CH}_{2} \mathrm{O}, \mathrm{CH}_{2} \mathrm{~N}, \mathrm{PhCH}_{2} \mathrm{~N}\right)$, $3.20\left(4 \mathrm{H}, \mathrm{t},{ }^{3} J=5.6 \mathrm{~Hz}, \mathrm{CH}_{2} \mathrm{NPh}\right), 6.44\left(2 \mathrm{H}, \mathrm{d}^{3}{ }^{3} J=7.2 \mathrm{~Hz}, \mathrm{H}(\mathrm{Ph})\right)$, $6.49\left(2 \mathrm{H}, \mathrm{d},{ }^{3} \mathrm{~J}=8.2 \mathrm{~Hz}, \mathrm{H}(\mathrm{Ph})\right), 6.85(2 \mathrm{H}$, br. s, H2(Ph)), $7.05(2 \mathrm{H}$, t, $\left.{ }^{3} J=7.8 \mathrm{~Hz}, \mathrm{H} 5(\mathrm{Ph})\right), 7.19\left(1 \mathrm{H}, \mathrm{d},{ }^{3} J=7.6 \mathrm{~Hz}, \mathrm{H} 6(\mathrm{Nf})\right), 7.49-7.60$ $(2 \mathrm{H}, \mathrm{m}, \mathrm{H} 3, \mathrm{H} 7(\mathrm{Nf})), 8.07\left(1 \mathrm{H}, \mathrm{d},{ }^{3} J=7.3 \mathrm{~Hz}, \mathrm{H} 2(\mathrm{Nf})\right), 8.41(1 \mathrm{H}$, br. d, $\left.{ }^{3} J_{\text {obs }}=8.3 \mathrm{~Hz}, \mathrm{H} 8(\mathrm{Nf})\right), 8.55\left(1 \mathrm{H}, \mathrm{d},{ }^{3} J=8.2 \mathrm{~Hz}, \mathrm{H} 4(\mathrm{Nf})\right)$, two $\mathrm{NH}$ protons were not unambiguously assigned. ${ }^{13} \mathrm{C} \mathrm{NMR}\left(\mathrm{CDCl}_{3}\right.$, $298 \mathrm{~K}) \delta_{c}$ ppm: 25.2 (3C, br. s, $\left.\Delta v_{1 / 2}=30 \mathrm{~Hz}, \mathrm{NCCH}_{2} \mathrm{CN}\right), 29.1$ (2C, $\left.\mathrm{NCCH}_{2} \mathrm{CO}\right), 41.4\left(2 \mathrm{C}, \mathrm{CH}_{2} \mathrm{NPh}\right), 45.3\left(2 \mathrm{C}, \mathrm{CH}_{3}\right), 46.9$ (2C, br. s, $\left.\Delta v_{1 / 2}=30 \mathrm{~Hz}, \mathrm{CH}_{2} \mathrm{~N}\right), 47.7\left(2 \mathrm{C}\right.$, br. s, $\left.\Delta v_{1 / 2}=30 \mathrm{~Hz}, \mathrm{CH}_{2} \mathrm{~N}\right)$, $48.5\left(2 \mathrm{C}\right.$, br. s, $\left.\Delta v_{1 / 2}=30 \mathrm{~Hz}, \mathrm{CH}_{2} \mathrm{~N}\right), 58.7\left(2 \mathrm{C}, \mathrm{PhCH}_{2} \mathrm{~N}\right), 69.6(2 \mathrm{C}$, $\left.\mathrm{CH}_{2} \mathrm{O}\right), 70.2\left(2 \mathrm{C}, \mathrm{CH}_{2} \mathrm{O}\right), 70.6\left(2 \mathrm{C}, \mathrm{CH}_{2} \mathrm{O}\right), 112.1(2 \mathrm{C}, \mathrm{CH}(\mathrm{Ph}))$, $113.6(2 \mathrm{C}, \mathrm{CH}(\mathrm{Ph})), 115.3(1 \mathrm{C}, \mathrm{CH}(\mathrm{Nf})), 117.7(2 \mathrm{C}, \mathrm{CH}(\mathrm{Ph})), 119.3$ (1C, $\mathrm{CH}(\mathrm{Nf})), 123.2$ (1C, CH(Nf)), 128.3 (1C, CH(Nf)), 129.4 (2C, $\mathrm{CH}(\mathrm{Ph})), 129.6(1 \mathrm{C}, \mathrm{CH}(\mathrm{Nf})), 130.1(1 \mathrm{C}, \mathrm{C}(\mathrm{Nf})), 130.3(1 \mathrm{C}, \mathrm{C}(\mathrm{Nf}))$, 130.7 (1C, CH(Nf)), 133.6 (1C, br. s, C(Nf)), 149.3 (2C, C3(Ph)), $151.9(1 \mathrm{C}, \mathrm{NC}(\mathrm{Nf}))$, quaternary carbon atom $\mathrm{Cl}(\mathrm{Ph})$ was not unambiguously assigned.

10-Phenyl-1,4,7-triazabicyclo[5.2.1]decane (14). ${ }^{[19]}$ A oneneck flask equipped with a magnetic stirrer was charged with 1,4,7-triazacyclononane ( $645 \mathrm{mg}, 5 \mathrm{mmol}), 120 \mathrm{ml}$ of ethanol, freshly distilled benzaldehyde $(0.5 \mathrm{ml}, 4.9 \mathrm{mmol})$ was added followed by molecular sieves, the mixture was stirred for $6 \mathrm{~h}$. The residue was filtered off, washed with ethanol $(10 \mathrm{ml})$, combined organic fractions were evaporated in vacuo to give compound $\mathbf{1 4}$ as pale-yellow crystals, m.p. $103-104{ }^{\circ} \mathrm{C}$. Yield $910 \mathrm{mg}(86 \%)$.

4 - (Naphthalen-2-ylmethyl)-10-phenyl-1,4,7triazabicyclo[5.2.1]decane (15). A one-neck flask equipped with a magnetic stirrer was charged with compound 14 (217 mg, $1 \mathrm{mmol})$, dissolved in $\mathrm{MeCN}(20 \mathrm{ml})$, 2-(bromomethyl) naphthalene $\left(221 \mathrm{mg}, 1 \mathrm{mmol}\right.$ ) was added followed by $\mathrm{K}_{2} \mathrm{CO}_{3}$
( $345 \mathrm{mg}, 2.5 \mathrm{mmol}$ ), the reaction was stirred for $24 \mathrm{~h}$, the residue was filtered off, washed with $\mathrm{CH}_{2} \mathrm{Cl}_{2}(10 \mathrm{ml})$, combined organic fractions were evaporated in vacuo to give compound 15 as a yellow oil. Yield $339 \mathrm{mg}$ (95\%). $\mathrm{m} / \mathrm{z}$ (MALDI-TOF) found: 358.2256. $\mathrm{C}_{24} \mathrm{H}_{28} \mathrm{~N}_{3}$ requires $358.2283[\mathrm{M}+\mathrm{H}]^{+}$. ${ }^{1} \mathrm{H}$ NMR $\left(\mathrm{CDCl}_{3}\right.$, $298 \mathrm{~K}) \delta_{\mathrm{H}}$ ppm: $2.74\left(2 \mathrm{H}, \mathrm{dt},{ }^{2} J=14.9 \mathrm{~Hz},{ }^{3} \mathrm{~J}=2.7 \mathrm{~Hz}, \mathrm{CH}_{2} \mathrm{~N}\right), 2.94$ $\left(2 \mathrm{H}, \mathrm{dt},{ }^{2} J=14.9 \mathrm{~Hz},{ }^{3} J=2.2 \mathrm{~Hz}, \mathrm{CH}_{2} \mathrm{~N}\right), 2.99-3.08\left(4 \mathrm{H}, \mathrm{m}, \mathrm{CH}_{2} \mathrm{~N}\right)$, $3.14-3.20\left(2 \mathrm{H}, \mathrm{m}, \mathrm{CH}_{2} \mathrm{~N}\right), 3.33-3.42\left(2 \mathrm{H}, \mathrm{m}, \mathrm{CH}_{2} \mathrm{~N}\right), 3.97(2 \mathrm{H}, \mathrm{s}$, $\left.\mathrm{CH}_{2} \mathrm{Nf}\right), 5.77(1 \mathrm{H}, \mathrm{s}, \mathrm{CHPh}), 7.31\left(1 \mathrm{H}, \mathrm{t},{ }^{3} J=7.4 \mathrm{~Hz}, \mathrm{H} 4(\mathrm{Ph})\right), 7.34$ $\left(2 \mathrm{H}, \mathrm{t},{ }^{3} \mathrm{~J}_{\text {obs }}=7.6 \mathrm{~Hz}, \mathrm{H} 3, \mathrm{H} 3\right.$ '(Ph)), 7.45-7.49 (2H, m, H(Nf)), 7.51 (1H, d, $\left.{ }^{3} J=8.1 \mathrm{~Hz}, \mathrm{H}(\mathrm{Nf})\right), 7.55-7.58$ (2H, m, H2, H2'(Ph)), 7.72 $(1 \mathrm{H}, \mathrm{s}, \mathrm{H} 1(\mathrm{Nf})), 7.80-7.85$ (3H, m, H(Nf). ${ }^{13} \mathrm{C} \mathrm{NMR}\left(\mathrm{CDCl}_{3}, 298\right.$ K) $\delta_{c}$ ppm: $49.2\left(2 \mathrm{C}, \mathrm{CH}_{2} \mathrm{~N}\right), 54.9\left(2 \mathrm{C}, \mathrm{CH}_{2} \mathrm{~N}\right), 56.3\left(2 \mathrm{C}, \mathrm{CH}_{2} \mathrm{~N}\right)$, $62.5\left(1 \mathrm{C}, \mathrm{CH}_{2} \mathrm{Nf}\right), 87.5$ (1C, CHPh), 125.5 (1C, $\left.\mathrm{CH}(\mathrm{Nf})\right), 125.9$ (1C, $\mathrm{CH}(\mathrm{Nf})), 126.4$ (3C, $\left.\mathrm{CH}(\mathrm{Ph}), \mathrm{C} 3, \mathrm{C}^{\prime}(\mathrm{Ph})\right), 126.8$ (1C, $\left.\mathrm{CH}(\mathrm{Nf})\right)$ 126.9 (1C, $\mathrm{CH}(\mathrm{Nf})), 127.6$ (2C, $\mathrm{CH}(\mathrm{Nf})), 127.9$ (2C, C2, C2'(Ph)), $128.0(1 \mathrm{C}, \mathrm{CH}(\mathrm{Nf})), 132.7(1 \mathrm{C}, \mathrm{C}(\mathrm{Nf})), 133.2(1 \mathrm{C}, \mathrm{C}(\mathrm{Nf})), 137.8$ $(1 \mathrm{C}, \mathrm{C} 2(\mathrm{Nf})), 145.4(1 \mathrm{C}, \mathrm{Cl}(\mathrm{Ph}))$.

1-(Naphthalenylmethyl)-1,4,7-triazacyclononane trihydrochloride (16). Synthesized from compound 15 (357 mg, $1 \mathrm{mmol}$ ) by stirring with $15 \mathrm{ml} 1 \mathrm{M} \mathrm{HCl}$ for $4 \mathrm{~h}$. The solution was evaporated in vacuo, the crystalline residue was washed with $10 \mathrm{ml}$ chloroform and dried in vacuo. White crystalline powder, yield $377 \mathrm{mg}$ (94\%). ${ }^{1} \mathrm{H}$ NMR ( $\left.\mathrm{D}_{2} \mathrm{O}, 298 \mathrm{~K}\right) \delta_{\mathrm{H}}$ ppm: $2.94-2.98$ $\left(4 \mathrm{H}, \mathrm{m}, \mathrm{CH}_{2} \mathrm{~N}\right), 3.11-3.15\left(4 \mathrm{H}, \mathrm{m}, \mathrm{CH}_{2} \mathrm{~N}\right), 3.57\left(4 \mathrm{H}, \mathrm{s}, \mathrm{CH}_{2} \mathrm{~N}\right), 3.95$ (2H, s, $\left.\mathrm{CH}_{2} \mathrm{Nf}\right), 7.48-7.55$ (3H, m, H(Nf)), 7.80 (1H, br.s, H1(Nf)), 7.85-7.90 (3H, s, H(Nf)). ${ }^{13} \mathrm{C}$ NMR $\left(\mathrm{D}_{2} \mathrm{O}, 298 \mathrm{~K}\right) \delta_{\mathrm{c}} \mathrm{ppm:} 42.2$ $\left(2 \mathrm{C}, \mathrm{CH}_{2} \mathrm{~N}\right), 43.6\left(2 \mathrm{C}, \mathrm{CH}_{2} \mathrm{~N}\right), 47.7\left(2 \mathrm{C}, \mathrm{CH}_{2} \mathrm{~N}\right), 59.2\left(1 \mathrm{C}, \mathrm{CH}_{2} \mathrm{Nf}\right)$, $126.6(1 \mathrm{C}, \mathrm{CH}(\mathrm{Nf})), 126.7$ (1C, CH(Nf)), 127.7 (1C, CH(Nf)), 127.9 (2C, $\mathrm{CH}(\mathrm{Nf})), 128.4(1 \mathrm{C}, \mathrm{CH}(\mathrm{Nf})), 129.2(1 \mathrm{C}, \mathrm{CH}(\mathrm{Nf})), 132.6(1 \mathrm{C}$, $\mathrm{C}(\mathrm{Nf})), 132.8$ (1C, C(Nf)), 132.9 (1C, C(Nf)).

1-(Naphthalen-2-ylmethyl)-1,4,7-triazacyclononane

(17). Synthesized from compound $\mathbf{1 6}$ (377 $\mathrm{mg}, 0.94 \mathrm{mmol}$ ) by stirring with $\mathrm{NaOH}(109 \mathrm{mg}, 2.72 \mathrm{mmol})$ in $5 \mathrm{ml}$ water for 2 min followed by extraction with chloroform $(4 \times 50 \mathrm{ml})$, combined organic fractions were evaporated in vacuo to obtain yellow-brown glassy compound. Yield $216 \mathrm{mg}$ (86 \%). $\mathrm{m} / \mathrm{z}$ (MALDI-TOF) found: 270.1935. $\mathrm{C}_{17} \mathrm{H}_{24} \mathrm{~N}_{3}$ requires $270.1970[\mathrm{M}+\mathrm{H}]^{+}$. ${ }^{1} \mathrm{H} \mathrm{NMR}\left(\mathrm{CDCl}_{3}\right.$, $298 \mathrm{~K}) \delta_{\mathrm{H}}$ ppm: $2.68\left(8 \mathrm{H}\right.$, br.s, $\left.\mathrm{CH}_{2} \mathrm{~N}\right), 2.82\left(4 \mathrm{H}\right.$, br.s, $\left.\mathrm{CH}_{2} \mathrm{~N}\right), 3.85$ $\left(2 \mathrm{H}, \mathrm{s}, \mathrm{CH}_{2} \mathrm{Nf}\right), 7.41-7.45(2 \mathrm{H}, \mathrm{m}, \mathrm{H}(\mathrm{Nf})), 7.50\left(1 \mathrm{H}, \mathrm{d},{ }^{3} J=8.5 \mathrm{~Hz}\right.$, $\mathrm{H}(\mathrm{Nf})), 7.68$ (1H, br.s, H1(Nf)), 7.77-7.82 (3H, m, H(Nf)), two NH protons were not assigned. ${ }^{13} \mathrm{C} \mathrm{NMR}\left(\mathrm{CDCl}_{3}, 298 \mathrm{~K}\right) \delta_{\mathrm{c}}$ ppm: 45.9 $\left(2 \mathrm{C}, \mathrm{CH}_{2} \mathrm{~N}\right), 46.1\left(2 \mathrm{C}, \mathrm{CH}_{2} \mathrm{~N}\right), 52.2\left(2 \mathrm{C}, \mathrm{CH}_{2} \mathrm{~N}\right), 61.7\left(1 \mathrm{C}, \mathrm{CH}_{2} \mathrm{Nf}\right)$, $125.6(1 \mathrm{C}, \mathrm{CH}(\mathrm{Nf})), 126.0(1 \mathrm{C}, \mathrm{CH}(\mathrm{Nf})), 127.2(1 \mathrm{C}, \mathrm{CH}(\mathrm{Nf})), 127.4$ (1C, $\mathrm{CH}(\mathrm{Nf})), 127.6(2 \mathrm{C}, \mathrm{CH}(\mathrm{Nf})), 128.1$ (1C, $\mathrm{CH}(\mathrm{Nf})), 132.7$ (1C, $\mathrm{C}(\mathrm{Nf})), 133.2(1 \mathrm{C}, \mathrm{C}(\mathrm{Nf})), 136.8(1 \mathrm{C}, \mathrm{C} 2(\mathrm{Nf}))$.

1,4-Bis(3-bromobenzyl)-7-(naphthalene-2-ylmethyl)1,4,7-triazacyclononane (18). A one-neck flask equipped with a magnetic stirrer was charged with compound 17 (200 mg, $0.743 \mathrm{mmol}$ ), dissolved in MeCN (4 ml), 3-bromobenzyl bromide $(280 \mathrm{mg}, 1.1 \mathrm{mmol})$ was added followed by $\mathrm{K}_{2} \mathrm{CO}_{3}(345 \mathrm{mg}$, $2.5 \mathrm{mmol}$ ), the reaction was stirred for $24 \mathrm{~h}$, the residue was filtered off, washed with $\mathrm{CH}_{2} \mathrm{Cl}_{2}(5 \mathrm{ml})$, combined organic fractions were evaporated in vacuo to give compound $\mathbf{1 8}$ as a yellow oil. Yield $301 \mathrm{mg}(90 \%) . \mathrm{m} / \mathrm{z}$ (MALDI-TOF) found: 606.1128. $\mathrm{C}_{31} \mathrm{H}_{34} \mathrm{Br}_{2} \mathrm{~N}_{3}$ requires $606.1119[\mathrm{M}+\mathrm{H}]^{+}$. ${ }^{1} \mathrm{H}$ NMR $\left(\mathrm{CDCl}_{3}, 298 \mathrm{~K}\right) \delta_{\mathrm{H}} \mathrm{ppm}$ : $2.78\left(4 \mathrm{H}\right.$, br.s, $\left.\mathrm{CH}_{2} \mathrm{~N}\right), 2.81\left(4 \mathrm{H}\right.$, br.s, $\left.\mathrm{CH}_{2} \mathrm{~N}\right), 2.86(4 \mathrm{H}$, br.s, $\left.\mathrm{CH}_{2} \mathrm{~N}\right), 3.57\left(4 \mathrm{H}, \mathrm{s}, \mathrm{NCH}_{2} \mathrm{Ph}\right), 3.80\left(2 \mathrm{H}\right.$, br.s, $\left.\mathrm{CH}_{2} \mathrm{Nf}\right), 7.15(2 \mathrm{H}, \mathrm{t}$, $\left.{ }^{3} J_{o b s}=7.8 \mathrm{~Hz}, \mathrm{H} 5(\mathrm{Ph})\right), 7.23\left(2 \mathrm{H}, \mathrm{d},{ }^{3} J=7.7 \mathrm{~Hz}, \mathrm{H} 6(\mathrm{Ph})\right), 7.37(2 \mathrm{H}$, d, $\left.{ }^{o b s}=7.8 \mathrm{~Hz}, \mathrm{H} 4(\mathrm{Ph})\right), 7.42-7.47(2 \mathrm{H}, \mathrm{m}, \mathrm{H}(\mathrm{Nf})), 7.49$ (2H, br.s, $\mathrm{H} 2(\mathrm{Ph})), 7.55$ (1H, d, $\left.{ }^{3} J=8.5 \mathrm{~Hz}, \mathrm{H}(\mathrm{Nf})\right), 7.69$ (1H, br.s, H1(Nf)), $7.78-7.84(3 \mathrm{H}, \mathrm{m}, \mathrm{H}(\mathrm{Nf})) .{ }^{13} \mathrm{C} \mathrm{NMR}\left(\mathrm{CDCl}_{3}, 298 \mathrm{~K}\right) \delta_{\mathrm{c}} \mathrm{ppm}: 55.2$ $\left(6 \mathrm{C}, \mathrm{br}, \mathrm{CH}_{2} \mathrm{~N}\right), 62.2\left(2 \mathrm{C}, \mathrm{PhCH}_{2} \mathrm{~N}\right), 63.0\left(1 \mathrm{C}, \mathrm{CH}_{2} \mathrm{Nf}\right), 122.3(2 \mathrm{C}$, $\mathrm{C} 3(\mathrm{Ph})), 125.6$ (1C, CH(Nf)), 125.9 (1C, CH(Nf)), 127.6 (6C, br, $\mathrm{CH}(\mathrm{Ph}), 4 \mathrm{CH}(\mathrm{Nf})), 127.8$ (1C, $\mathrm{CH}(\mathrm{Nf})), 129.7$ (2C, $\mathrm{CH}(\mathrm{Ph})), 129.9$ (2C, $\mathrm{CH}(\mathrm{Ph})), 131.9(2 \mathrm{C}, \mathrm{CH}(\mathrm{Ph})), 132.7$ (2C, $\mathrm{C}(\mathrm{Nf})), 133.2$ (1C, $\mathrm{C}(\mathrm{Nf})), 142.6(2 \mathrm{C}, \mathrm{Cl}(\mathrm{Ph}))$. 
37-(Naphthalen-2-ylmethyl)-10,13,16-trioxa-6,20-diaza3(1,4)-triazacyclononane-1,5(1,3)-dibenzenacycloicosaphane(19). Obtained according to a general procedure for macrocyclization from compound $\mathbf{1 8}(121 \mathrm{mg}, 0.2 \mathrm{mmol})$, trioxadiamine $\mathbf{1 0}$ (44 $\mathrm{mg}$, $0.2 \mathrm{mmol})$ in the presence of $\mathrm{Pd}(\mathrm{dba})_{2}(18 \mathrm{mg}, 0.032 \mathrm{mmol})$, DavePhos (14 mg, $0.036 \mathrm{mmol}), t \mathrm{BuONa}(77 \mathrm{mg}, 0.8 \mathrm{mmol})$ in $10 \mathrm{ml}$ of dioxane. Eluent: $\mathrm{CH}_{2} \mathrm{Cl}_{2}-\mathrm{MeOH} 5: 1$, yield $7 \mathrm{mg}$ (6\%), yellow glassy solid. $\mathrm{m} / z$ (MALDI-TOF) found: 666.4360 . $\mathrm{C}_{41} \mathrm{H}_{56} \mathrm{~N}_{5} \mathrm{O}_{3}$ requires $666.4383[\mathrm{M}+\mathrm{H}]^{+} .{ }^{1} \mathrm{H} \mathrm{NMR}\left(\mathrm{CDCl}_{3}, 298 \mathrm{~K}\right) \delta_{\mathrm{H}}$ ppm: $1.83\left(4 \mathrm{H}\right.$, quintet, $\left.{ }^{3} \mathrm{~J}=5.7 \mathrm{~Hz}, \mathrm{CCH}_{2} \mathrm{C}\right), 2.85\left(4 \mathrm{H}\right.$, br.s, $\left.\mathrm{CH}_{2} \mathrm{~N}\right)$, 3.08 (4H, br.s, $\left.\mathrm{CH}_{2} \mathrm{~N}\right), 3.27\left(4 \mathrm{H}, \mathrm{t},{ }^{3} \mathrm{~J}=5.9 \mathrm{~Hz}, \mathrm{CH}_{2} \mathrm{NPh}\right), 3.45-3.75$ $\left(18 \mathrm{H}, \mathrm{m}, \mathrm{CH}_{2} \mathrm{O}, \mathrm{CH}_{2} \mathrm{~N}\right), 3.82\left(2 \mathrm{H}\right.$, br.s, $\left.\mathrm{NfCH}_{2} \mathrm{~N}\right), 6.35-6.82(4 \mathrm{H}$, $\mathrm{m}, \mathrm{H}(\mathrm{Ph})), 6.62\left(2 \mathrm{H}, \mathrm{d},{ }^{3} J=8.2 \mathrm{~Hz}, \mathrm{H}(\mathrm{Ph})\right), 7.15\left(2 \mathrm{H}, \mathrm{t},{ }^{3} J=7.8 \mathrm{~Hz}\right.$, H5(Ph)), 7.45-7.52 (3H, m, H(Nf)), 7.66 (1H, s, H1(Nf)), 7.79-7.83 $(3 \mathrm{H}, \mathrm{m}, \mathrm{H}(\mathrm{Nf}))$, two $\mathrm{NH}$ protons were not assigned.

9-((1,4,7-Triazacyclononan-1-yl)methyl)acridine (21). A one-neck flask equipped with a magnetic stirrer was charged with compound 14 (54 mg, $0.25 \mathrm{mmol})$, dissolved in $\mathrm{MeCN}$ (6 ml), 9-(bromomethyl)acridine ( $68 \mathrm{mg}, 0.25 \mathrm{mmol}$ ) was added followed by $\mathrm{K}_{2} \mathrm{CO}_{3}(86 \mathrm{mg}, 0.625 \mathrm{mmol}$ ), the reaction was stirred for $24 \mathrm{~h}$, the residue was filtered off, washed with $\mathrm{CH}_{2} \mathrm{Cl}_{2}(5 \mathrm{ml})$, combined organic fractions were evaporated in vacuo and the residue was chromatographed on silica gel. Eluent: $\mathrm{CH}_{2} \mathrm{Cl}_{2}-\mathrm{MeOH}-\mathrm{NH}_{3}$ 100:20:2, yield $42 \mathrm{mg}$ (52\%), yellow glassy solid. $\mathrm{m} / z$ (MALDITOF) found: 321.2135. $\mathrm{C}_{20} \mathrm{H}_{25} \mathrm{~N}_{4}$ requires $321.2079[\mathrm{M}+\mathrm{H}]^{+} .{ }^{1} \mathrm{H}$ NMR $\left(\mathrm{CDCl}_{3}, 298 \mathrm{~K}\right) \delta_{\mathrm{H}} \mathrm{ppm}^{2} 2.54\left(4 \mathrm{H}, \mathrm{t},{ }^{3} \mathrm{~J}=5.5 \mathrm{~Hz}, \mathrm{CH}_{2} \mathrm{~N}\right)$, $2.72\left(4 \mathrm{H}, \mathrm{s}, \mathrm{CH}_{2} \mathrm{~N}\right), 2.75\left(4 \mathrm{H}, \mathrm{t},{ }^{3} \mathrm{~J}=5.5 \mathrm{~Hz}, \mathrm{CH}_{2} \mathrm{~N}\right), 4.34(2 \mathrm{H}$, br.s, NH), $4.64\left(2 \mathrm{H}, \mathrm{s}, \mathrm{AcrCH}_{2} \mathrm{~N}\right), 7.56-7.60(2 \mathrm{H}, \mathrm{m}, \mathrm{H}(\mathrm{Acr}))$, 7.70-7.73 (2H, m, H(Acr)), 8.18 (2H, d, ${ }^{3} J=8.7 \mathrm{~Hz}, \mathrm{H} 1, \mathrm{H} 8($ Acr $)$ ), $8.39\left(2 \mathrm{H}, \mathrm{d},{ }^{3} \mathrm{~J}=8.8 \mathrm{~Hz}, \mathrm{H} 4, \mathrm{H} 5(\mathrm{Acr})\right) \cdot{ }^{13} \mathrm{C} \mathrm{NMR}\left(\mathrm{CDCl}_{3}, 298 \mathrm{~K}\right)$ $\delta_{\mathrm{c}}$ ppm: $44.3\left(2 \mathrm{C}, \mathrm{CH}_{2} \mathrm{~N}\right), 45.3\left(2 \mathrm{C}, \mathrm{CH}_{2} \mathrm{~N}\right), 51.8\left(2 \mathrm{C}, \mathrm{CH}_{2} \mathrm{~N}\right), 52.1$ $\left(1 \mathrm{C}, \mathrm{NfCH}_{2} \mathrm{~N}\right), 124.2$ (2C, $\left.\mathrm{CH}(\mathrm{Acr})\right), 125.7$ (2C, C(Ar)), $126.4(2 \mathrm{C}$, CH(Acr)), 129.8 (2C, CH(Acr)), 130.3 (2C, CH(Acr)), 140.4 (1C, C9(Acr)), 148.5 (2C, C(Acr)).

1,4,7-Tris(acridin-9-ylmethyl)-1,4,7-triazacyclononane (23). Obtained as the second product in the synthesis of compound 21. Eluent: $\mathrm{CH}_{2} \mathrm{Cl}_{2}-\mathrm{MeOH} 10: 1$, yield $20 \mathrm{mg}$ (34\%), yellow glassy solid. $m / z$ (MALDI-TOF) found: 703.3588. $\mathrm{C}_{48} \mathrm{H}_{43} \mathrm{~N}_{6}$ requires $703.3549[\mathrm{M}+\mathrm{H}]^{+} .{ }^{1} \mathrm{H} \mathrm{NMR}\left(\mathrm{CDCl}_{3}, 298 \mathrm{~K}\right) \delta_{\mathrm{H}} \mathrm{ppm}: 2.65(12 \mathrm{H}$, $\left.\mathrm{s}, \mathrm{CH}_{2} \mathrm{~N}\right), 4.25\left(6 \mathrm{H}, \mathrm{s}, \mathrm{AcrCH}_{2} \mathrm{~N}\right), 7.44-7.47(2 \mathrm{H}, \mathrm{m}, \mathrm{H}(\mathrm{Acr}))$, 7.71-7.75 (2H, m, H(Acr)), 8.20 (2H, d, ${ }^{3} J=8.7 \mathrm{~Hz}, \mathrm{H} 1, \mathrm{H} 8$ (Acr)), $8.30\left(2 \mathrm{H}, \mathrm{d},{ }^{3} J=8.8 \mathrm{~Hz}, \mathrm{H} 4, \mathrm{H} 5(\mathrm{Acr})\right) .{ }^{13} \mathrm{C} \mathrm{NMR}\left(\mathrm{CDCl}_{3}, 298 \mathrm{~K}\right) \delta_{\mathrm{c}}$ ppm: $53.5\left(3 \mathrm{C}, \mathrm{AcrCH}_{2} \mathrm{~N}\right), 55.4\left(6 \mathrm{C}, \mathrm{CH}_{2} \mathrm{~N}\right), 125.1(2 \mathrm{C}, \mathrm{CH}(\mathrm{Acr}))$, 125.6 (2C, $\mathrm{CH}(\mathrm{Acr})), 125.9$ (2C, C(Acr)), 129.7 (2C, $\mathrm{CH}(\mathrm{Acr}))$, 130.1 (2C, CH(Acr)), 142.0 (1C, C9(Acr)), 148.6 (2C, C(Acr)).

9-((4,7-Bis(3-bromobenzyl)-1,4,7-triazacyclononan-1-yl) methyl)acridine (24). A one-neck flask equipped with a magnetic stirrer was charged with compound 21 ( $53 \mathrm{mg}, 0.166 \mathrm{mmol})$, dissolved in $\mathrm{MeCN}$ (3 ml), 3-bromobenzyl bromide $(50 \mathrm{mg}, 0.2 \mathrm{mmol})$ was added followed by $\mathrm{K}_{2} \mathrm{CO}_{3}(138 \mathrm{mg}, 1 \mathrm{mmol})$, the reaction was stirred for $24 \mathrm{~h}$, the residue was filtered off, washed with $\mathrm{CH}_{2} \mathrm{Cl}_{2}$ $(5 \mathrm{ml})$, combined organic fractions were evaporated in vacuo to give compound 24 as a yellow oil. Yield $79 \mathrm{mg}(72 \%) . \mathrm{m} / z$ (MALDITOF) found: 657.1176. $\mathrm{C}_{34} \mathrm{H}_{35} \mathrm{Br}_{2} \mathrm{~N}_{4}$ requires $657.1228[\mathrm{M}+\mathrm{H}]^{+} .{ }^{1} \mathrm{H}$ NMR $\left(\mathrm{CDCl}_{3}, 298 \mathrm{~K}\right) \delta_{\mathrm{H}}$ ppm: $2.63\left(4 \mathrm{H}, \mathrm{s}, \mathrm{CH}_{2} \mathrm{~N}\right), 2.63-2.68(4 \mathrm{H}$, m, $\left.\mathrm{CH}_{2} \mathrm{~N}\right), 2.83-2.88\left(4 \mathrm{H}, \mathrm{m}, \mathrm{CH}_{2} \mathrm{~N}\right), 3.38\left(4 \mathrm{H}, \mathrm{s}, \mathrm{PhCH}_{2} \mathrm{~N}\right), 4.50$ $\left(2 \mathrm{H}, \mathrm{s}, \mathrm{AcrCH}_{2} \mathrm{~N}\right), 7.10\left(4 \mathrm{H}, \mathrm{d},{ }^{3} \mathrm{~J}_{o b s}=5.2 \mathrm{~Hz}, \mathrm{H} 4, \mathrm{H} 6(\mathrm{Ph})\right), 7.30-7.34$ (2H, m, H5(Ph)), 7.37 (2H, br.s, H2(Ph)), 7.52-7.56 (2H, m, H(Acr)), 7.73-7.76 (2H, m, H(Acr)), 8.22 (2H, d, ${ }^{3} J=8.3 \mathrm{~Hz}, \mathrm{H} 1, \mathrm{H} 8(\mathrm{Acr})$ ), $8.47\left(2 \mathrm{H}, \mathrm{d},{ }^{3} \mathrm{~J}=8.8 \mathrm{~Hz}, \mathrm{H} 4, \mathrm{H} 5(\mathrm{Acr})\right) .{ }^{13} \mathrm{C} \mathrm{NMR}\left(\mathrm{CDCl}_{3}, 298 \mathrm{~K}\right)$

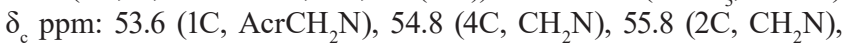
$62.0\left(2 \mathrm{C}, \mathrm{PhCH}_{2} \mathrm{~N}\right), 122.2(2 \mathrm{C},(\mathrm{C} 3(\mathrm{Ph})), 125.1(2 \mathrm{C}, \mathrm{CH}(\mathrm{Acr})), 125.7$ (2C, $\mathrm{CH}(\mathrm{Acr})), 125.9$ (2C, C(Acr)), 127.4 (2C, $\mathrm{CH}(\mathrm{Ph})), 129.7$ (4C, $\mathrm{CH}(\mathrm{Ph})), 129.9$ (2C, $\mathrm{CH}(\mathrm{Acr})), 130.1$ (2C, $\mathrm{CH}(\mathrm{Acr}))$, 137. 2 (2C, $\mathrm{CH}(\mathrm{Ph})), 142.0$ (1C, C9(Acr)), 142.4 (2C, C1(Ph)), 148.6 (2C, C(Acr)). 37-(Acridin-9-ylmethyl)-10,13,16-trioxa-6,20-diaza-3(1,4)triazacyclononane-1,5(1,3)-dibenzenacycloicosaphane
Obtained according to a general procedure for macrocyclization from compound 24 (79 mg, $0.12 \mathrm{mmol})$, trioxadiamine 10 (27 mg, $0.12 \mathrm{mmol})$ in the presence of $\mathrm{Pd}(\mathrm{dba})_{2}(11 \mathrm{mg}, 0.0192 \mathrm{mmol})$, DavePhos (9 mg, $0.0216 \mathrm{mmol}$ ), $t \mathrm{BuONa}$ (46 mg, $0.48 \mathrm{mmol}$ ) in $8 \mathrm{ml}$ dioxane. Eluent: $\mathrm{CH}_{2} \mathrm{Cl}_{2}-\mathrm{MeOH} 5: 1$, yield $8 \mathrm{mg}(9 \%)$, yellow glassy solid. $\mathrm{m} / \mathrm{z}$ (MALDI-TOF) found: 717.4547. $\mathrm{C}_{44} \mathrm{H}_{57} \mathrm{~N}_{6} \mathrm{O}_{3}$ requires $717.4492[\mathrm{M}+\mathrm{H}]^{+} .{ }^{1} \mathrm{H}$ NMR $\left(\mathrm{CDCl}_{3}, 298 \mathrm{~K}\right)$ $\delta_{\mathrm{H}}$ ppm: $1.82\left(4 \mathrm{H}\right.$, quintet, $\left.{ }^{3} \mathrm{~J}=5.8 \mathrm{~Hz}, \mathrm{CCH}_{2} \mathrm{C}\right), 2.60-3.90(28 \mathrm{H}$, $\left.\mathrm{m}, \mathrm{CH}_{2} \mathrm{~N}, \mathrm{CH}_{2} \mathrm{O}\right), 3.21\left(4 \mathrm{H}, \mathrm{t},{ }^{3} \mathrm{~J}=5.9 \mathrm{~Hz}, \mathrm{CH}_{2} \mathrm{NNPh}\right), 4.72 \mathrm{~s}(2 \mathrm{H}$, s, $\left.\mathrm{AcrCH}_{2} \mathrm{~N}\right), 6.30-6.75(6 \mathrm{H}, \mathrm{m}, \mathrm{H}(\mathrm{Ph})), 7.11(2 \mathrm{H}$, br.s, H5(Ph)), 7.65-7.70 (2H, m, H(Acr)), 7.78-7.83 (2H, m, H(Acr)), $8.26(2 \mathrm{H}$, d, $\left.{ }^{3} J=8.3 \mathrm{~Hz}, \mathrm{H} 1, \mathrm{H} 8(\mathrm{Acr})\right), 8.49$ (2H, d, ${ }^{3} J=8.1 \mathrm{~Hz}, \mathrm{H} 4, \mathrm{H} 5$ (Acr)), two NH protons were not assigned.

1,4,7-Tris(3-bromobenzyl)-1,4,7-triazacyclononane (26). A one-neck flask equipped with a magnetic stirrer was charged with TACN (129 mg, $1 \mathrm{mmol})$, dissolved in MeCN ( $8 \mathrm{ml})$, 3-bromobenzyl bromide (750 mg, $3 \mathrm{mmol}$ ) was added followed by $\mathrm{K}_{2} \mathrm{CO}_{3}(690 \mathrm{mg}$, $5 \mathrm{mmol}$ ), the reaction was stirred for $24 \mathrm{~h}$, the residue was filtered off, washed with $\mathrm{CH}_{2} \mathrm{Cl}_{2}(10 \mathrm{ml})$, combined organic fractions were evaporated in vacuo to give compound $\mathbf{2 6}$ as a colourless oil. Yield $623 \mathrm{mg}(98 \%) . \mathrm{m} / z$ (MALDI-TOF) found: 634.0124. $\mathrm{C}_{27} \mathrm{H}_{31} \mathrm{Br}_{3} \mathrm{~N}_{3}$ requires $634.0068[\mathrm{M}+\mathrm{H}]^{+}$. ${ }^{1} \mathrm{H} \mathrm{NMR}\left(\mathrm{CDCl}_{3}, 298 \mathrm{~K}\right) \delta_{\mathrm{H}} \mathrm{ppm:} 2.77$ $\left(12 \mathrm{H}, \mathrm{s}, \mathrm{CH}_{2} \mathrm{~N}\right), 3.56\left(6 \mathrm{H}, \mathrm{s}, \mathrm{PhCH}_{2} \mathrm{~N}\right), 7.16\left(3 \mathrm{H}, \mathrm{t},{ }^{3} \mathrm{~J}=7.7 \mathrm{~Hz}\right.$, $\mathrm{H} 5(\mathrm{Ph})), 7.24\left(3 \mathrm{H}, \mathrm{d},{ }^{3} \mathrm{~J}=7.6 \mathrm{~Hz}, \mathrm{H} 6(\mathrm{Ph})\right), 7.35\left(3 \mathrm{H}, \mathrm{d},{ }^{3} J=7.8 \mathrm{~Hz}\right.$, $\mathrm{H} 4(\mathrm{Ph})), 7.48$ (3H, br.s, H2(Ph)). ${ }^{13} \mathrm{C} \mathrm{NMR}\left(\mathrm{CDCl}_{3}, 298 \mathrm{~K}\right) \delta_{\mathrm{c}} \mathrm{ppm}$ : $55.3\left(6 \mathrm{C}, \mathrm{CH}_{2} \mathrm{~N}\right), 62.3\left(3 \mathrm{C}, \mathrm{PhCH}_{2} \mathrm{~N}\right), 122.3(3 \mathrm{C}, \mathrm{C} 3(\mathrm{Ph})), 127.5$ (3C, $\mathrm{CH}(\mathrm{Ph})), 129.7(3 \mathrm{C}, \mathrm{CH}(\mathrm{Ph})), 129.9$ (3C, $\mathrm{CH}(\mathrm{Ph})), 131.8$ (3C, $\mathrm{CH}(\mathrm{Ph})), 142.7(3 \mathrm{C}, \mathrm{Cl}(\mathrm{Ph}))$.

1,4,7-Tris(3-(1,4,7,10-tetraoxa-13-azacyclopentadecan13-yl)benzyl)-1,4,7-triazacyclononane (28). A two-necked flask flushed with argon was charged with compound 26 (127 mg, $0.2 \mathrm{mmol})$, 1-aza-15-crown-5 ether (27) (131 mg, $0.6 \mathrm{mmol})$, $\mathrm{Pd}(\mathrm{dba})_{2}$ (28 mg, $\left.0.048 \mathrm{mmol}\right)$, DavePhos (21 mg, $\left.0.054 \mathrm{mmol}\right)$, dioxane $(1 \mathrm{ml})$ was added followed by $t \mathrm{BuONa}(86 \mathrm{mg}, 0.9 \mathrm{mmol})$. The reaction mixture was stirred at reflux for $24 \mathrm{~h}$, then cooled down to ambient temperature, diluted with $5 \mathrm{ml}$ dichloromethane, the residue was filtered off, washed with $5 \mathrm{ml}$ dichloromethane, the combined organic fractions were evaporated in vacuo and the residue was chromatographed on silica gel. Eluent: $\mathrm{CH}_{2} \mathrm{Cl}_{2}-\mathrm{MeOH}-\mathrm{NH}_{3}$ 100:20:3, yield $25 \mathrm{mg}$ (12\%), pale-yellow glassy solid. $m / z$ (MALDI-TOF) found: 1051.6774. $\mathrm{C}_{57} \mathrm{H}_{91} \mathrm{~N}_{6} \mathrm{O}_{12}$ requires $1051.6695[\mathrm{M}+\mathrm{H}]^{+} .{ }^{1} \mathrm{H} \mathrm{NMR}\left(\mathrm{CDCl}_{3}, 298 \mathrm{~K}\right) \delta_{\mathrm{H}} \mathrm{ppm:} 2.87$ $\left(12 \mathrm{H}\right.$, br.s, $\left.\mathrm{CH}_{2} \mathrm{~N}\right), 3.57\left(12 \mathrm{H}, \mathrm{t},{ }^{3} \mathrm{~J}=6.1 \mathrm{~Hz}, \mathrm{CH}_{2} \mathrm{NPh}\right), 3.58-3.67$ $\left(42 \mathrm{H}, \mathrm{m}, \mathrm{CH}_{2} \mathrm{O}, \mathrm{PhCH}_{2} \mathrm{~N}\right), 3.72\left(12 \mathrm{H}, \mathrm{t},{ }^{3} \mathrm{~J}=6.1 \mathrm{~Hz}, \mathrm{CH}_{2} \mathrm{O}\right), 6.55$ (6H, br.d, $\left.{ }^{3} \mathrm{~J}_{\text {obs }}=7.2 \mathrm{~Hz}, \mathrm{H} 4, \mathrm{H} 6(\mathrm{Ph})\right), 6.69$ (3H, br.s, H2(Ph)), 7.12 $\left(3 \mathrm{H}, \mathrm{t},{ }^{3} J=7.8 \mathrm{~Hz}, \mathrm{H} 5(\mathrm{Ph})\right) \cdot{ }^{13} \mathrm{C}$ NMR $\left(\mathrm{CDCl}_{3}, 298 \mathrm{~K}\right) \delta_{\mathrm{c}} \mathrm{ppm}: 52.5$ $\left(6 \mathrm{C}, \mathrm{CH}_{2} \mathrm{NPh}\right), 54.5\left(6 \mathrm{C}, \mathrm{br}, \Delta \mathrm{v}_{1 / 2}=200 \mathrm{~Hz}, \mathrm{CH}_{2} \mathrm{~N}\right), 62.8(3 \mathrm{C}, \mathrm{br}$, $\left.\Delta \mathrm{v}_{1 / 2}=60 \mathrm{~Hz}, \mathrm{PhCH}_{2} \mathrm{~N}\right), 68.6\left(6 \mathrm{C}, \mathrm{CH}_{2} \mathrm{O}\right), 70.0\left(6 \mathrm{C}, \mathrm{CH}_{2} \mathrm{O}\right), 70.1$ $\left(6 \mathrm{C}, \mathrm{CH}_{2} \mathrm{O}\right), 71.2\left(6 \mathrm{C}, \mathrm{CH}_{2} \mathrm{O}\right), 110.5(3 \mathrm{C}, \mathrm{CH}(\mathrm{Ph})), 112.3(3 \mathrm{C}$, $\mathrm{CH}(\mathrm{Ph})), 116.6(3 \mathrm{C}, \mathrm{CH}(\mathrm{Ph})), 129.2(3 \mathrm{C}, \mathrm{C} 5(\mathrm{Ph})), 147.8$ (3C, $\mathrm{C} 3(\mathrm{Ph}))$. Quaternary carbon atoms $\mathrm{Cl}(\mathrm{Ph})$ were not assigned.

$1,4,11,14,17,24,33$-Heptaazahexacyclo[12.12.9.24,24 $\cdot 1^{6,10} \cdot 1^{18,22}$. $\left.1^{28,32}\right]$ tetraconta-6(40), 7,9,18(39),19,21,28(36),29,31-nonane (30). Obtained according to a general procedure for macrocyclization from compound $\mathbf{2 6}$ (127 mg, $0.2 \mathrm{mmol})$, tris(2-aminoethyl)amine (29) $(27 \mathrm{mg}, 0.12 \mathrm{mmol})$ in the presence of $\mathrm{Pd}(\mathrm{dba})_{2}(28 \mathrm{mg}$, $0.048 \mathrm{mmol}$ ), DavePhos (21 mg, $0.054 \mathrm{mmol}), t \mathrm{BuONa}(86 \mathrm{mg}$, $0.9 \mathrm{mmol})$ in $10 \mathrm{ml}$ of dioxane. Eluent: $\mathrm{CH}_{2} \mathrm{Cl}_{2}-\mathrm{MeOH} 2: 1$, yield $15 \mathrm{mg}$ (14\%), yellow oil. $\mathrm{m} / \mathrm{z}$ (MALDI-TOF) found: 540.3854 . $\mathrm{C}_{33} \mathrm{H}_{46} \mathrm{~N}_{7}$. requires $540.3815[\mathrm{M}+\mathrm{H}]^{+} .{ }^{1} \mathrm{H} \mathrm{NMR}\left(\mathrm{CDCl}_{3}, 298 \mathrm{~K}\right) \delta_{\mathrm{H}}$ ppm: $2.95\left(12 \mathrm{H}\right.$, br.s, $\left.\mathrm{CH}_{2} \mathrm{~N}\right), 3.16\left(12 \mathrm{H}\right.$, br.s, $\left.\mathrm{CH}_{2} \mathrm{~N}\right), 3.54(6 \mathrm{H}, \mathrm{s}$, $\left.\mathrm{PhCH}_{2} \mathrm{~N}\right), 4.97$ (3H, br.s, NH), 6.07 (3H, br.s, H2(Ph)), $6.54(3 \mathrm{H}$, $\left.\mathrm{d},{ }^{3} J=7.7 \mathrm{~Hz}, \mathrm{H}(\mathrm{Ph})\right), 6.59\left(3 \mathrm{H}, \mathrm{d},{ }^{3} J=7.3 \mathrm{~Hz}, \mathrm{H}(\mathrm{Ph})\right), 7.18(3 \mathrm{H}, \mathrm{t}$, $\left.{ }^{3} J=7.7 \mathrm{~Hz}, \mathrm{H} 5(\mathrm{Ph})\right) .{ }^{13} \mathrm{C} \mathrm{NMR}\left(\mathrm{CDCl}_{3}, 298 \mathrm{~K}\right) \delta_{\mathrm{c}} \mathrm{ppm}: 40.9(3 \mathrm{C}$, $\left.\mathrm{CH}_{2} \mathrm{NPh}\right), 48.5\left(6 \mathrm{C}, \mathrm{CH}_{2} \mathrm{~N}\right), 50.0\left(3 \mathrm{C}, \mathrm{CH}_{2} \mathrm{~N}\right), 60.6\left(3 \mathrm{C}, \mathrm{PhCH}_{2} \mathrm{~N}\right)$, $110.2(3 \mathrm{C}, \mathrm{CH}(\mathrm{Ph})), 116.1$ (3C, $\mathrm{CH}(\mathrm{Ph})), 120.6$ (3C, $\mathrm{CH}(\mathrm{Ph})), 129.9$ (3C, C5(Ph)), $135.2(3 \mathrm{C}, \mathrm{Cl}(\mathrm{Ph})), 148.1(3 \mathrm{C}, \mathrm{C} 3(\mathrm{Ph}))$. 
The investigations of the spectral properties of the bicycles 11-13 in the presence of 20 metal salts were carried out in a following manner: $3 \mathrm{ml}$ of the solution of the corresponding bicycle $(C=21.9 \mu \mathrm{M}$ for $\mathbf{1 1}, 21.1 \mu \mathrm{M}$ for $12,15.9 \mu \mathrm{M}$ for 13) in $\mathrm{MeCN}$ were placed in a spectrofluorimetric cuvette, solutions of appropriate metal salts ( $\mathrm{Li}(\mathrm{I}), \mathrm{Na}(\mathrm{I}), \mathrm{K}(\mathrm{I}), \mathrm{Mg}(\mathrm{II}), \mathrm{Ca}(\mathrm{II})$, $\mathrm{Ba}(\mathrm{II}), \mathrm{Al}(\mathrm{III}), \mathrm{Fe}(\mathrm{II}), \mathrm{Mn}(\mathrm{II}), \mathrm{Co}(\mathrm{II}), \mathrm{Ni}(\mathrm{II}), \mathrm{Cr}(\mathrm{III}), \mathrm{Cu}(\mathrm{II})$, $\mathrm{Zn}(\mathrm{II}), \mathrm{Cd}(\mathrm{II}), \mathrm{Pb}(\mathrm{II}), \mathrm{Hg}(\mathrm{II})$ perchlorates and $\mathrm{Ga}(\mathrm{III}), \mathrm{In}(\mathrm{III})$, $\mathrm{Y}(\mathrm{III})$ nitrates) in $\mathrm{MeCN}(C=0.01 \mathrm{M})$ were added sequentially (1, $2,5,10$ equiv.) and after each addition UV-Vis and fluorescence spectra were recorded.

\section{Results and Discussion}

For the synthesis of bicyclic derivatives of triazacycloalkanes we intended to modify them with one fluorophore group at the first step and with two bromobenzyl groupsatthesecondstep fortheaccomplishmentofthecatalytic macrocyclization at the final step. Our investigation started from the synthesis of $N$-dansyl substituted TACN and TACD. For this purpose starting free triazacycloalkanes $\mathbf{1}$ and $\mathbf{2}$ were reacted with dansyl chloride in $\mathrm{MeCN}$ at room temperature in the presence of $\mathrm{K}_{2} \mathrm{CO}_{3}$ (Scheme 1). To ensure a sufficient yield of the monodansylated products $\mathbf{3}$ and $\mathbf{4}$ the following precautions are to be taken into consideration: 1) the application of no more than $0.7-0.75$ equiv. of dansyl chloride is important to diminish the formation of diand tridansyl substituted by-products; 2) enough diluted solution of dansyl chloride $(C=0.01 \mathrm{M})$ should be added very slowly during several hours to a solution of compounds $\mathbf{1}$ or $\mathbf{2}$ to prevent an easy formation of indicated by-products; 3) only extra-pure $\mathrm{MeCN}$ should be used to exclude the traces of acrylonitrile which readily reacts with TACN and TACD under reaction conditions diminishing the yields of the target products; 4) only chloroform can be used for chromatographic isolation of compound $\mathbf{3}$ as it readily reacts with dichloromethane forming bicyclic aminal.

In the case of TACN the yield of compound 3 was $68 \%$ while the $N, N$ '-didansyl derivative 5 was isolated in $29 \%$ yield. However, TACD was prone for polysubstitution even in the presence of 0.7 equiv. of dansyl chloride, thus the yield of the target product was only $28 \%$ and the second product 6 was obtained in $65 \%$ yield. All compounds were isolated by column chromatography on silica gel. Further reactions of compound 3 with 3- and 4-bromobenzyl bromides proceeded smoothly affording corresponding bis(bromobenzyl) substituted TACN 7 and $\mathbf{8}$ in high yields, but the reaction of TACD derivative $\mathbf{4}$ with 3 -bromobenzyl bromide was complicated with the formation of mono- and tribenzylated by-products what led to a necessity of chromatographic separation and gave the desired compound in $38 \%$ yield.

Catalytic macrocyclization reactions of compound $\mathbf{7}$ was first carried out with linear trioxadiamine $\mathbf{1 0}$ using a standard protocol in the presence of a traditional $\mathrm{Pd}(\mathrm{dba})_{2} /$ BINAP (dba=dibenzylideneacetone, BINAP=2,2'-bis(diphenylphosphino)-1,1'-binaphthalene) system (Scheme 2). The yield of the macrocycle $\mathbf{1 1}$ was $30 \%$ in the reaction mixture and after column chromatography it was isolated in $16 \%$ yield. The change of BINAP for a more electron-rich phosphine ligand, i.e. DavePhos (2-(dicyclohexylphosphino)2'-dimethylaminobiphenyl) did not notably increased the yield in the reaction mixture (32\%) but helped to improve the result after chromatographic isolation (24\%) probably due to a change in the composition of by-products which affect the efficacy of chromatography. The reaction of the same trioxadiamine with bis(4-bromobenzyl) derivative 8 catalyzed by $\mathrm{Pd}(\mathrm{dba})_{2} /$ DavePhos system provided $27 \%$ yield of compound $\mathbf{1 2}$ in the reaction mixture and $15 \%$ yield after its separation. Supposedly, the macrocyclization processes involving di(3-bromobenzyl) substituted precursors are generally more successful than those with isomeric di(4bromobenzyl) derivatives, probably due to a longer intramolecular distance between two reaction centers in the latter case. This fact was earlier observed in our works. ${ }^{[20]}$ Macrocyclization with TACD derivative 9 was the most successful as it provided $26 \%$ yield of the macrobicycle $\mathbf{1 3}$ after isolation.

${ }^{1} \mathrm{H}$ NMR spectra of compounds 11, 12 registered in $\mathrm{CDCl}_{3}$ at $298 \mathrm{~K}$ possess a very broad multiplet in 3.0-4.0 ppm region which is associated with $\mathrm{CH}_{2}$ protons in triazacyclononane ring and in benzyl groups. Signals of some other aromatic protons in benzyl spacers are also notably broadened contrary to aromatic protons in dansyl fluorophore. Analogously, in ${ }^{13} \mathrm{C}$ NMR spectra the signals of corresponding carbon atoms are also more or less

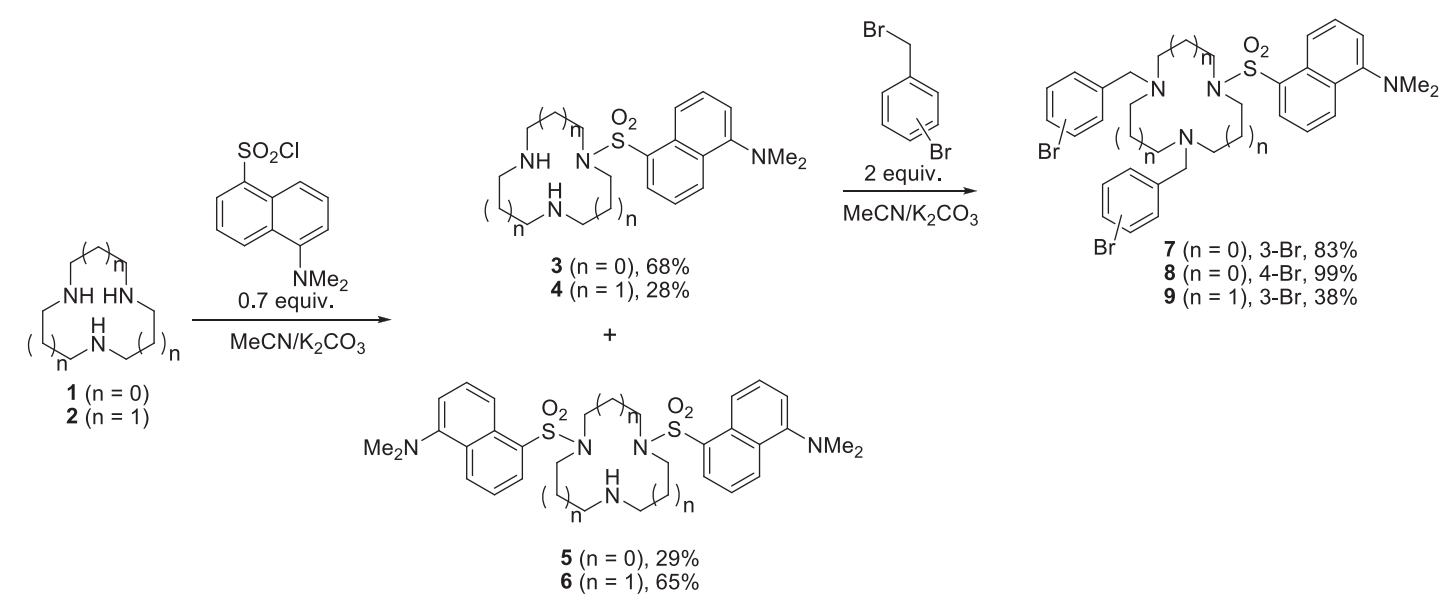

Scheme 1. 
broadened. The attempt to sharpen the signals by a change of $\mathrm{CDCl}_{3}$ for DMSO- $d_{6}$ leads to even more broadening of all signals at $298 \mathrm{~K}$, but at $363 \mathrm{~K}$ some of them become narrow, however it is insufficient to obtain fully resolved signals for all protons. These facts can be explained by high-energy conformational transitions in these bicyclic compounds which were not observed for previously obtained macrobicycles based on dibenzyl substituted diazacrown ethers, ${ }^{[21]}$ cyclen or cyclam $^{[22]}$ but were noted for the derivatives of tetrabenzyl substituted cyclen and cyclam. ${ }^{[23]}$

The approach which was found to be useful for the introduction of one dansyl group in TACN molecule could not be applied for the modification of this triazacycle with other fluorophore groups like naphthalene and acridine. The reactions of TACN with various amounts of 2-(bromomethyl)and 2-(chloromethyl)naphthalene (0.5-1 equiv.) yielded inseparable mixtures of mono-, di- and trisubstituted TACN. To overcome this difficulty, we tried the method of TACN functionalization using the aminal protecting group. ${ }^{[19]}$ According to a described method a bicyclic compound 14 was obtained in a high yield by reacting free TACN with benzaldehyde (Scheme 3). The reaction of compound 14 with 1 equiv. 2-(bromomethyl)naphthalene under standard conditions gave compound $\mathbf{1 5}$ in $95 \%$ yield. After treatment by $\mathrm{HCl}$ in water to remove the aminal protection and washing with chloroform, trihydrochloride form $\mathbf{1 6}$ was obtained in $94 \%$ yield. Its neutralization with $\mathrm{KOH}$ and extraction with chloroform provided $86 \%$ yield of the monosubstitued TACN 17. Its reaction with 2 equiv. 3-bromobenzyl bromide proceeded smoothly affording trisubstituted TACN 18 (90\% yield), and the macrocyclization was carried out using trioxadiamine $\mathbf{1 0}$ in the presence of $\mathrm{Pd}(\mathrm{dba})_{2} / \mathrm{DavePhos}$ system. The yield of the target bicycle 19 after preparative chromatography was however quite poor $(6 \%)$.

The possibility to carry out a multistep synthesis of the macrobicycle containing a fluorophore group which cannot be introduced directly gave grounds for the synthesis of the relative compound possessing another fluorophore, i.e. acridine. The reaction of bicyclic aminal 14 with 9-(bromomethyl)acridine unexpectedly resulted in the formation of a mixture of deprotected mono-, di- and trisubstituted derivatives 21, 22 and 23 in which compounds 21 and 23 prevailed (Scheme 4). Surprisingly, the expected monoacridinyl derivative with the aminal protection $\mathbf{2 0}$ was observed
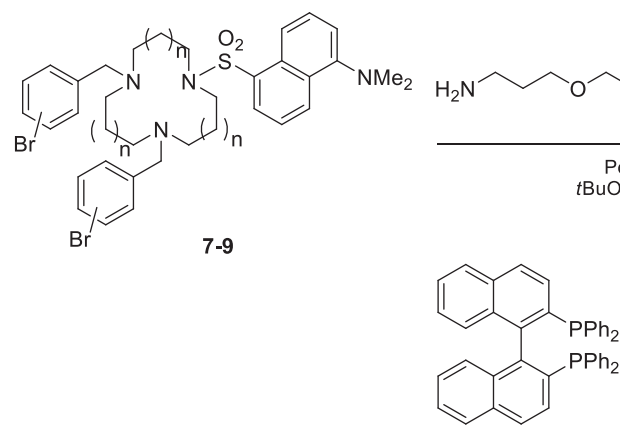

rac-BINAP

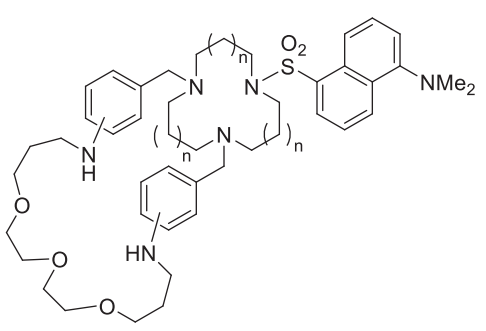

$11(\mathrm{n}=0), m-\mathrm{NH} ; 16 \%$ ( $\mathrm{L}=\mathrm{BINAP}), 24 \%$ ( $\mathrm{L}=$ DavePhos $)$ $13(\mathrm{n}=1), m-\mathrm{NH} ; 26 \%$ ( $(\mathrm{L}=$ = DavePhos $)$

Scheme 2.
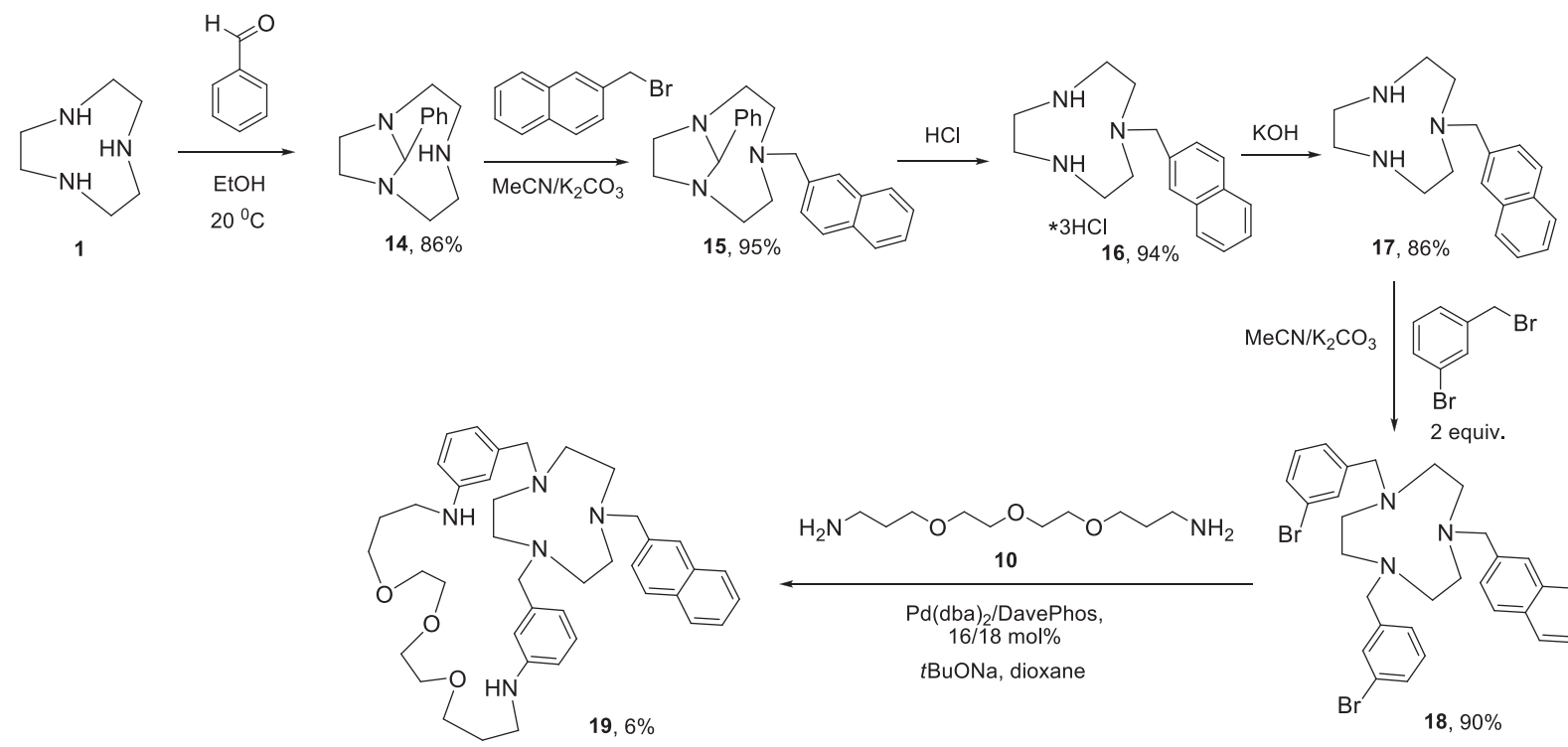

$t$ BuONa, dioxane

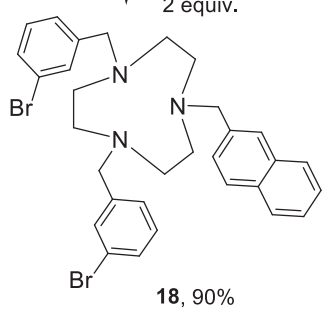

Scheme 3. 
in the reaction mixture only in trace amounts (by $m / z=409$ $[\mathrm{M}+\mathrm{H}]^{+}$in the MALDI-TOF spectrum and by a characteristic singlet at $5.65 \mathrm{ppm}$ in the ${ }^{1} \mathrm{H}$ NMR spectrum which corresponds to $\mathrm{CHN}_{2}$ proton). Chromatographic separation of the reaction mixture afforded compounds $\mathbf{2 1}$ and $\mathbf{2 3}$ in 52 and $34 \%$ yields, respectively. The reaction of 21 with 2 equiv. 3-bromobenzyl bromide gave trisubstituted TACN derivative $\mathbf{2 4}$ in $\mathbf{7 2} \%$ yield. The macrocyclization reaction of this compound with trioxadiamine $\mathbf{1 0}$ catalyzed by $\mathrm{Pd}(\mathrm{dba})_{2} /$ DavePhos system gave rise to the target macrocycle $\mathbf{2 5}$ in $9 \%$, however, it contained some admixtures which could not be separated by chromatography.

The ability of TACN to easily form trialkyl derivatives was employed in the synthesis of its tris(3-bromobenzyl) derivative $\mathbf{2 6}$ which was obtained in nearly quantitative yield (Scheme 5). The compound $\mathbf{2 6}$ was introduced in the $\operatorname{Pd}(0)$-catalyzed amination with 3 equiv. 1-aza15-crown-5 (27) in the presence of $\mathrm{Pd}(\mathrm{dba})_{2} /$ DavePhos system ( $8 \mathrm{~mol} \%$ catalyst per each amino group), and desired tetramacrocyclic compound $\mathbf{2 8}$ was isolated in $12 \%$ yield. The majority of starting compound was converted into biand tricyclic derivatives due to $\mathrm{C}-\mathrm{Br}$ bond catalytic reduction. The compound $\mathbf{2 6}$ was shown to participate in the catalytic "end-capping" reaction with tris(2-aminoethyl)amine (29) which allowed the synthesis of a tetracyclic cryptand $\mathbf{3 0}$ in $14 \%$ yield (Scheme 5).

The dansyl fluorophore group present in bicycles 11-13 is responsible for the absorption band at $340 \mathrm{~nm}$<smiles>c1ccccc1</smiles>

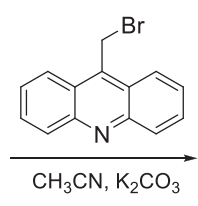

14

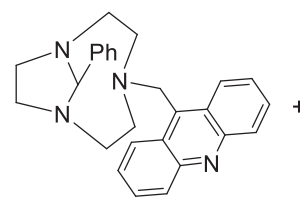

20 , observed in the reaction mixture

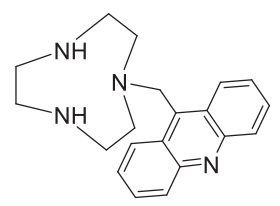

21, $52 \%$

$24,72 \%$

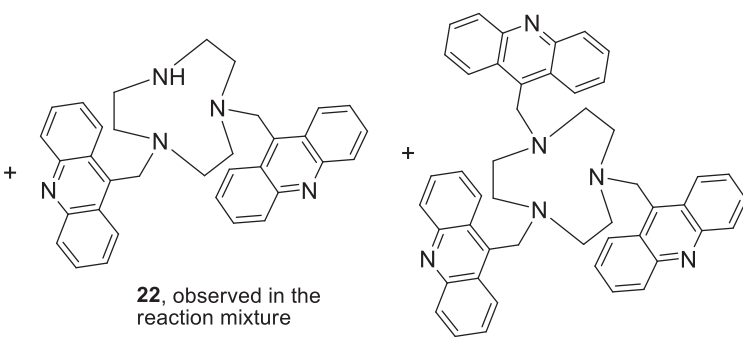

$23,34 \%$

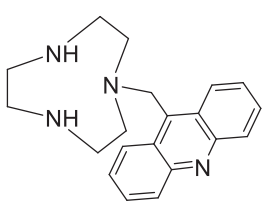

21

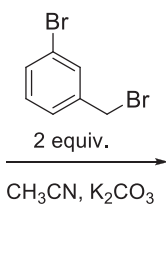

$$
\mathrm{Br}
$$<smiles>Brc1cccc(CN2CCN(Cc3cccc(Br)c3)CCN(Cc3c4ccccc4nc4ccccc34)CCN(Cc3cccc(Br)c3)CC2)c1</smiles>

$\mathrm{H}_{2} \mathrm{~N} \sim \mathrm{O} \sim \mathrm{O} \sim \mathrm{O} \sim \mathrm{NH}_{2}$<smiles></smiles>

Scheme 4 .

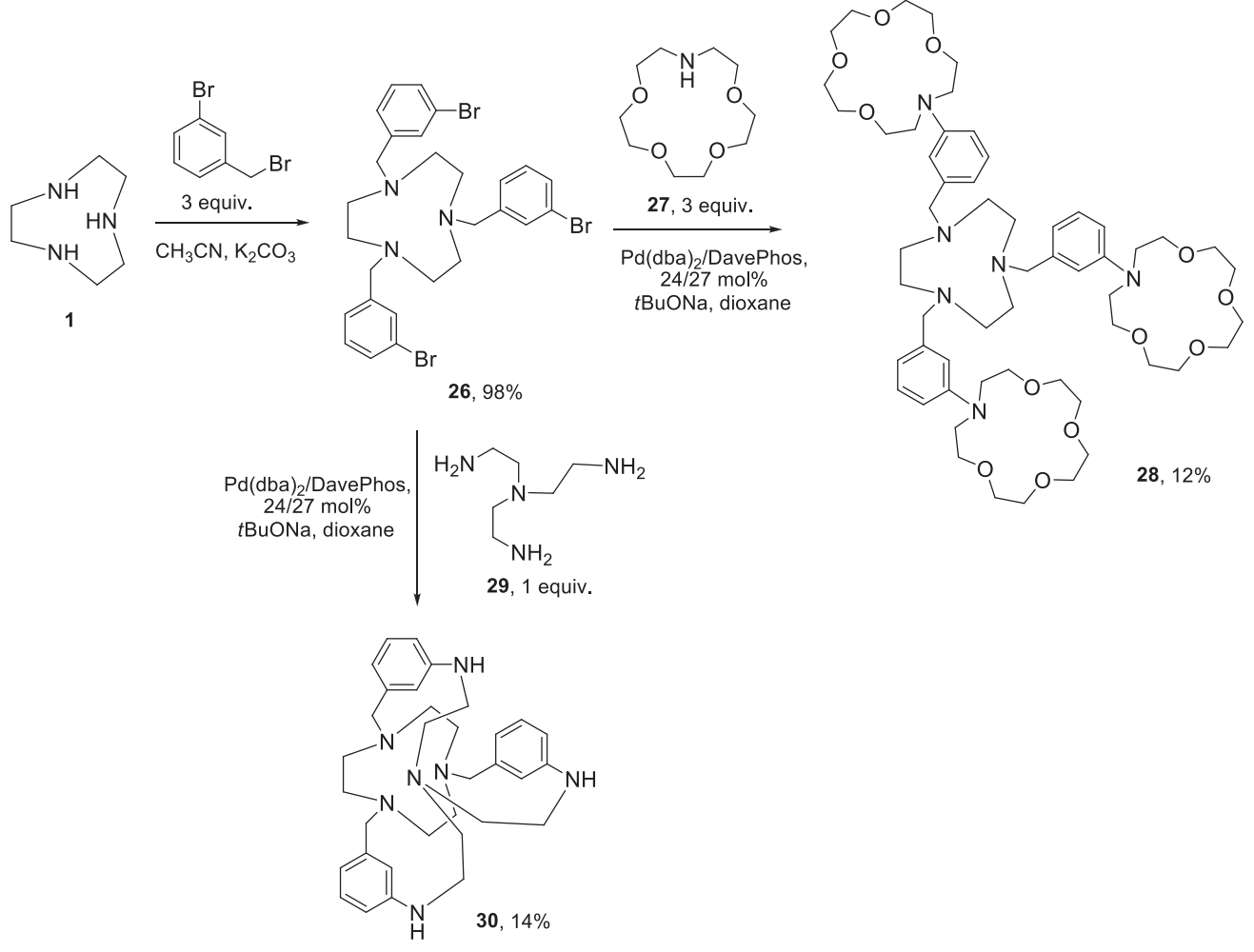

Scheme 5. 
and intensive fluorescence at $c a 520 \mathrm{~nm}$. We investigated the possibilities of these compounds to work as fluorescent chemosensors for metal cations. In the course of investigation UV-Vis and fluorescent spectra of these compounds were recorded in $\mathrm{MeCN}$ in the presence of 1, 2, 5 equiv. of corresponding metal perchlorates or nitrates: $\mathrm{Li}(\mathrm{I}), \mathrm{Na}(\mathrm{I})$, $\mathrm{K}(\mathrm{I}), \mathrm{Mg}(\mathrm{II}), \mathrm{Ca}(\mathrm{II}), \mathrm{Ba}(\mathrm{II}), \mathrm{Al}(\mathrm{III}), \mathrm{Mn}(\mathrm{II}), \mathrm{Fe}(\mathrm{II}), \mathrm{Co}(\mathrm{II})$, $\mathrm{Ni}(\mathrm{II}), \mathrm{Cu}(\mathrm{II}), \mathrm{Zn}(\mathrm{II}), \mathrm{Cd}(\mathrm{II}), \mathrm{Hg}(\mathrm{II}), \mathrm{Ag}(\mathrm{I}), \mathrm{Pb}(\mathrm{II})$ (perchlorates) and Ga(III), In(III), Y(III) (nitrates). The addition of 5 equiv. of corresponding cations to the cryptand $\mathbf{1 1}$ did not result in notable changes in the emission intensity except for $\mathrm{Cu}(\mathrm{II})$ and $\mathrm{Al}(\mathrm{III})$ which totally quenched fluorescence (Figure S1). These changes in fluorescence were accompanied with substantial changes in its UV-Vis spectra (Figure S2) as the addition of both metals led to disappearance of the absorption maximum at $305 \mathrm{~nm}$ and gave rise to a new maximum at $290 \mathrm{~nm}$ in the case of Al(III). Macrobicycles 12 and $\mathbf{1 3}$ were found to respond in a similar manner to the addition of 20 above mentioned metals (Figures S3 and S4) with almost total emission quenching in the presence of $\mathrm{Al}(\mathrm{III})$ and $\mathrm{Cu}$ (II). In the case of these cryptands we also noted $40-50 \%$ reduction of the fluorescence intensity in the presence of 5 equiv. Ga(III) and In(III), as well as a slight emission enhancement in the presence of $\mathrm{K}(\mathrm{I})$ together with a small bathochromic shift of the maximum (from 520 to $513 \mathrm{~nm}$ ). One may conclude that the emission quenching in the presence of $\mathrm{Al}(\mathrm{III})$ and $\mathrm{Cu}(\mathrm{II})$ is caused by the coordination with the dansyl fluorophore groups, probably via dimethylamino groups. There are literature data on a similar quenching of emission in dansylated azacrown ethers by $\mathrm{Cu}(\mathrm{II}), \mathrm{Al}(\mathrm{III})$ and $\mathrm{Pb}$ (II) cations. ${ }^{[24]} \mathrm{We}$ suppose that in the present case $\mathrm{Pb}$ (II) did not led to equal diminishing in fluorescence intensity due to a different coordination mode with $\mathrm{N}$ and $\mathrm{O}$ atoms of the bicyclic structures.

\section{Conclusions}

To sum up, we developed the synthetic approach to previously unknown bicyclic derivatives of 1,4,7-triazacyclononane (TACN) and 1,5,9-triazacyclododecane (TACD) bearing dansyl, naphthalene and acridine fluorophore groups using $\mathrm{Pd}(0)$-catalyzed amination reactions, demonstrated the possibility to use unprotected TACN and TACD in the synthesis of dansyl-substituted bicycles, obtained tetracyclic derivatives of TACN using its tris(3bromobenzyl) derivative, and showed the possibility to use dansylated bicycles as fluorescent molecular probes for detecting $\mathrm{Cu}(\mathrm{II})$ and $\mathrm{Al}(\mathrm{III})$ cations.
Acknowledgements. This work was financially supported by the RFBR grant N 17-53-16012.

\section{References}

1. Kumar M., Kumar N., Bhalla V., Singh H., Sharma P.R., Kaur T. Org. Lett. 2011, 13, 1422.

2. Ho I.-T., Haung K.-Ch., Chung W.-Sh. Chem. Asian. J. 2011, 6, 2738.

3. Kumar M., Kumar N., Bhalla V. Dalton Trans. 2012, 41, 10189.

4. Li M., Lu H.-Y., Liu R.-L., Chen J.-D., Chen Ch.-F. J. Org. Chem. 2012, 77, 3670.

5. Mewis R.E., Archibald S.J. Coord. Chem. Rev. 2010, 254, 1686.

6. Simecek J., Zemek O., Hermann P., Wester H.-J., Notni J. ChemMedChem 2012, 7, 1375.

7. Fujiwara M., Matsushita T., Wakita H. Anal. Sci. 1991, 7, 321.

8. Barreto J., Venkatachalam T.K., Joshi T., Krehe U., Forsyth C.M., Reutens D., Spiccia L. Polyhedron 2013, 52, 128.

9. Zeng Z., Torriero A.A.J., Bond A.M., Spiccia L. Chem. Eur. J. 2010, 16, 9154.

10. Lee M.H., Kim H.J., Yoon S., Park N., Kim J.S. Org. Lett. 2008, 10, 213.

11. Tharamaraj V., Pitchumani K. Anal. Chim. Acta 2012, 751, 171.

12. Metivier R., Leray I., Valeur B. Chem. Eur. J. 2004, 10, 4480.

13. Roper E.D., Talonov V.S., Gorbunova M.G. Anal. Chem. 2007, 79, 1983.

14. Chen Q.-Y., Chen C.-F. Tetrahedron Lett. 2005, 46, 165.

15. Averin A.D., Uglov A.N., Beletskaya I.P. Chem. Lett. 2008, 37, 1074.

16. Averin A.D., Shukhaev A.V., Buryak A.K., Denat F., Guilard R., Beletskaya I.P. Tetrahedron Lett. 2008, 49, 3950.

17. Yakushev A.A., Averin A.D., Maloshitskaya O.A., Syrbu S.A., Koifman O.I., Beletskaya I.P. Mendeleev Commun. 2016, 26, 199.

18. Ukai T., Kawazura H., Ishii Y., Bonnet J.J., Ibers J.A. J. Organomet. Chem. 1974, 65, 253.

19. Roger M., Patinec V., Bourgeois M., Tripier R., Triki S., Handel H. Tetrahedron 2012, 68, 5637.

20. Uglov A.N., Zubrienko G.A., Abel A.S., Averin A.D., Maloshitskaya O.A., Bessmertnykh-Lemeune A., Denat F., Beletskaya I.P. Heterocycles 2014, 88, 1213.

21. Yakushev A.A., Chernichenko N.M., Anokhin M.V., Averin A.D., Buryak A.K., Denat F., Beletskaya I.P. Molecules 2014, 19, 940.

22. Kobelev S.M., Averin A.D., Buryak A.K., Denat F., Guilard R., Beletskaya I.P. Heterocycles 2011, 82, 1447.

23. Kobelev S.M., Averin A.D., Buryak A.K., Vovk A.I., Kukhar V.P., Denat F., Guilard R., Beletskaya I.P. Heterocycles 2014, 90, 989.

24. Warmke H., Wiczk W., Ossowski T. Talanta 2000, 52, 449. 